\title{
EVALUATING REMAPPED PHYSICAL REACH FOR HAND INTERACTIONS WITH PASSIVE HAPTICS IN VIRTUAL REALITY
}

\author{
A Thesis \\ by \\ DUSTIN THO HAN
}
Submitted to the Office of Graduate and Professional Studies of Texas A\&M University in partial fulfillment of the requirements for the degree of

\section{MASTER OF SCIENCE}

Chair of Committee, Eric D. Ragan

Committee Members, Tim McLaughlin

Eric Jing Du

Head of Department, Tim McLaughlin

December 2017

Major Subject: Visualization

Copyright 2017 Dustin Tho Han 


\begin{abstract}
Virtual reality applications often make use of motion tracking to incorporate physical hand movements into interaction techniques for selection and manipulation techniques of virtual objects. To increase realism and allow direct hand interaction, real-world physical objects can be aligned with virtual objects to provide tactile feedback and physical grasping. However, unless a physical space is custom configured to match a specific virtual reality experience, the ability to perfectly match the physical and virtual objects is limited. Our research addresses this challenge by studying methods that allow one physical object to be mapped to multiple virtual objects that can exist as different virtual locations in an egocentric reference frame. We study two such techniques: one that introduces a static translational offset between the virtual and physical hand before a hand reach, and one that dynamically interpolates the position of the virtual hand during a reaching motion. We conducted two controlled experiments to assess how the two techniques affect reaching effectiveness, comfort, and ability to adapt to the remapping techniques when reaching for objects with different types of mismatches between physical and virtual locations. In addition, we present a case study to demonstrate how the hand remapping techniques could be used in an immersive game application to support realistic hand interaction while optimizing usability. With our results, we discuss future considerations for how to best implement passive haptics with remapping techniques and provide guidelines for effective implementation.
\end{abstract}




\section{ACKNOWLEDGMENTS}

I would like to thank my committee chair, Dr. Eric Ragan, for the constant feedback, support, and criticism. Thank you for keeping me on track and spending endless hours of meetings with me drawing on white boards and discussing my research. I would also like to thank my committee members, Tim McLaughlin and Dr. Eric Jing Du for their support.

My friends have played a huge role in helping me complete this thesis as they are constantly their to keep my morale and motivation up and for that I am always grateful. I do not believe there is anyone who could get through graduate school without the support of friends and I am no exception.

I am especially thankful for my loving family especially my parents who constantly remind me that they are my biggest fans. Without them, I would not even be here at Texas A\&M University with the opportunity to pursue my dreams. Thank you for always believing in me despite my personal doubts.

Finally, I would like to thank Texas A\&M University and the Department of Visualization for providing me with this opportunity and experience to learn and grow which I do not expect to end after graduation. 


\section{CONTRIBUTORS AND FUNDING SOURCES}

\section{Contributors}

This work was supported by a thesis committee consisting of Dr. Eric D. Ragan and Tim McLaughlin of the Department of Visualization and Dr. Eric Jing Du of the Department of Construction Science. All work conducted for the thesis was completed by the student independently.

\section{Funding Sources}

No outside funding was received for this graduate study. 


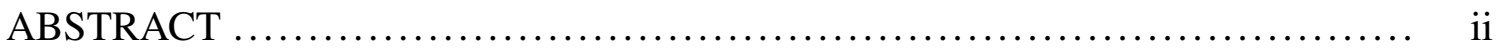

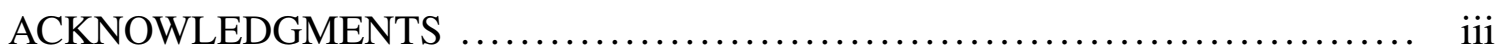

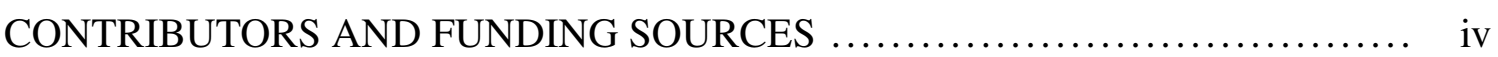

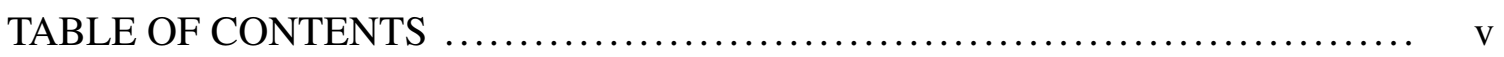

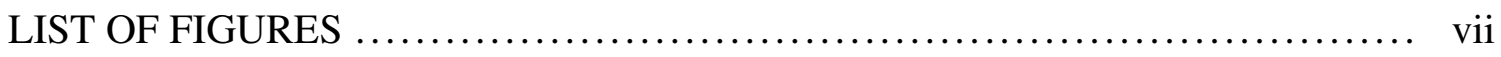

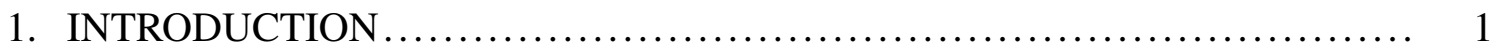

1.1 Motivation $\ldots \ldots \ldots \ldots \ldots \ldots \ldots \ldots \ldots \ldots \ldots \ldots \ldots \ldots \ldots \ldots \ldots \ldots \ldots \ldots, \quad 1$

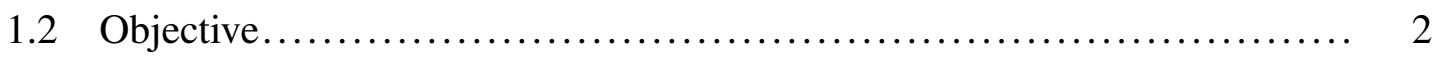

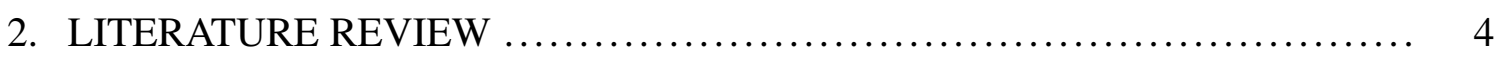

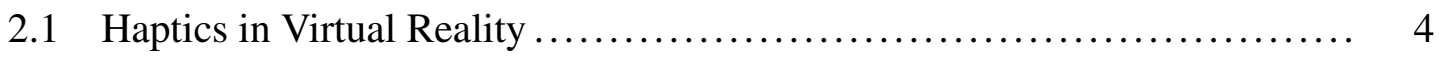

2.2 Visual and Proprioceptive Mismatch .............................. 5

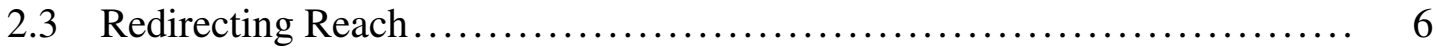

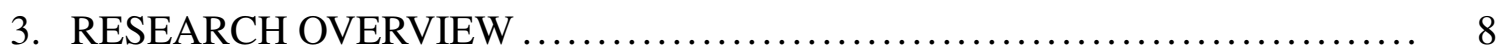

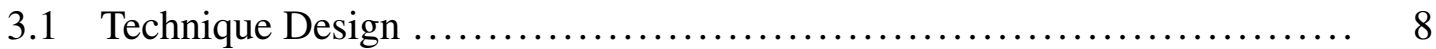

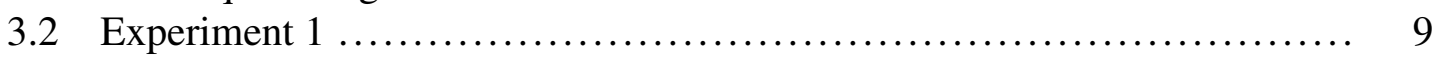

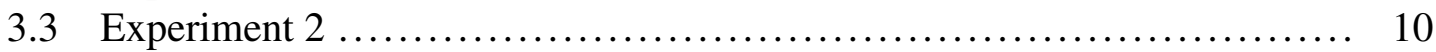

3.4 Case Study .................................................. 10

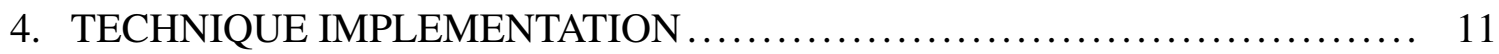

4.1 Materials ..................................................... 11

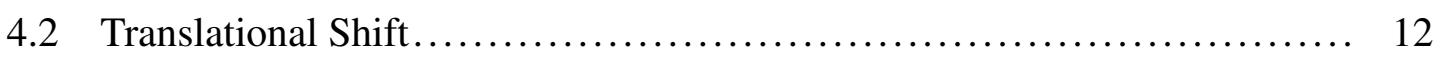

4.3 Interpolated Reach ....................................... 13

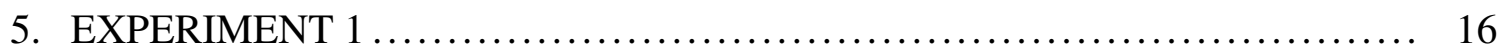

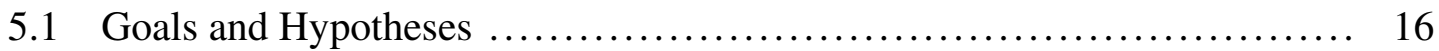

5.2 Experimental Task and Environment............................ 16 
5.3 Experimental Design ......................................... 18

5.4 Procedure ..................................................... 21

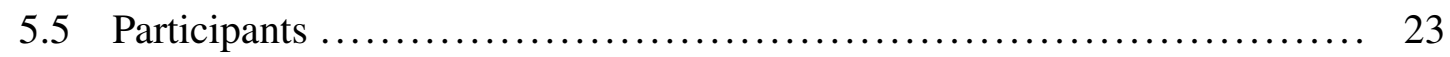

5.6 Results and Discussion....................................... 23

5.6.1 Quantitative Results ................................... 25

5.6 .2 Qualitative Feedback ................................ 30

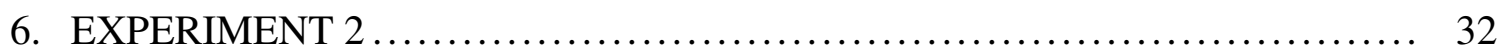

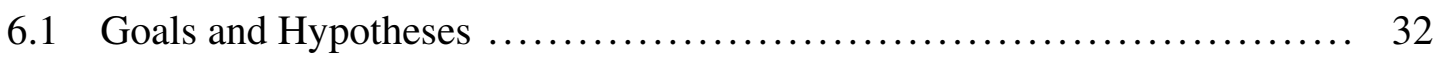

6.2 Experimental Task and Environment ............................ 32

6.3 Experimental Design ......................................... 33

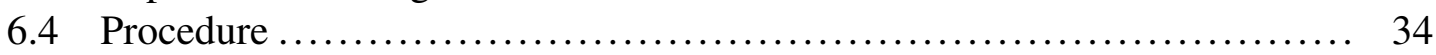

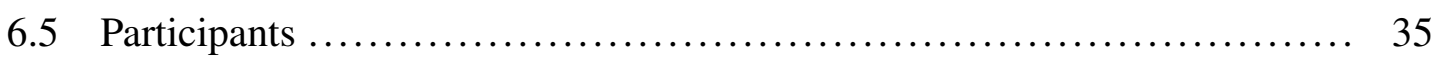

6.6 Results and Discussion........................................ 35

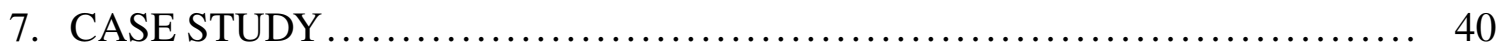

7.1 Goals ...................................................... 40

7.2 Game Application ........................................... 40

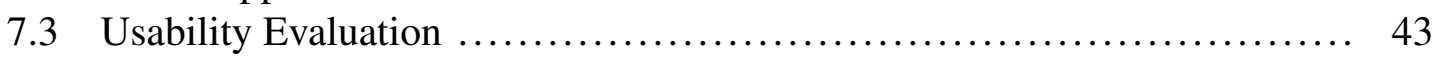

7.4 Results and Design Guidelines ................................. 44

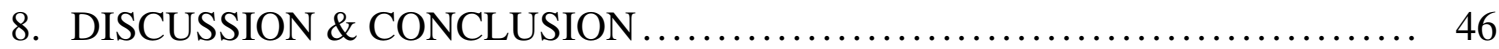

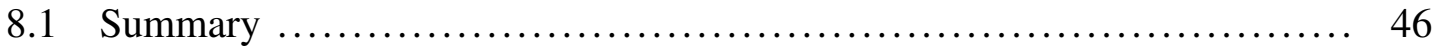

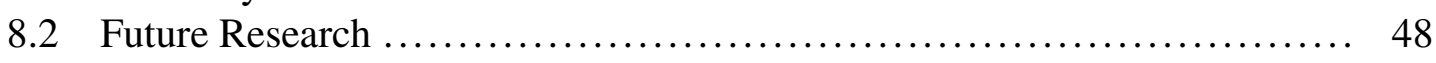

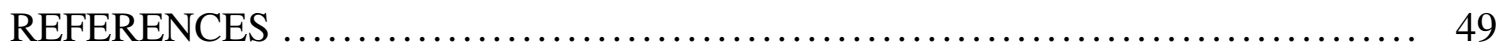

APPENDIX A. EXPERIMENT DOCUMENTS $\ldots \ldots \ldots \ldots \ldots \ldots \ldots \ldots \ldots \ldots \ldots \ldots \ldots \ldots$ 


\section{LIST OF FIGURES}

FIGURE

4.1 The physical setup used for the experiments includes a table, a stationary chair, a tracked bottle, and hand markers, and an HMD.

4.2 A top-down diagram demonstrating translational shift.

4.3 A top-down diagram demonstrating interpolated reach.

5.1 An image of the experimental environments shows how a green "GO" sign was presented to the participant to signal them to reach the green cylindrical virtual object and place it on the red target...

5.2 A total of 17 table positions were used in Experiment 1 marked in this image as black dots...

5.3 Regions for object distance.

5.4 Examples of offset directions

5.5 Reach times from Experiment 1 separated by technique type, offset direction, and offset size.

5.6 Errors from Experiment 1 separated by technique type, offset direction, and offset size.

5.7 Average reach times and errors from Experiment 1 based on table position, where the shape of the data table corresponds to the shape of the physical table, with the user's location represented by the gray circle.

6.1 Results for Experiment 2 showing reach time over 30 tasks for translational shift configurations.

6.2 Results for Experiment 2 showing reach time over 30 tasks for inteprolated reach configurations.

7.1 The virtual hand is offset to map to the virtual object highlighted in green based on user gaze. 
7.2 Monster combat scenario

A.1 Background Questionnaire Page $1 \ldots \ldots \ldots \ldots \ldots \ldots \ldots \ldots \ldots \ldots \ldots \ldots \ldots \ldots \ldots$

A.2 Background Questionnaire Page $2 \ldots \ldots \ldots \ldots \ldots \ldots \ldots \ldots \ldots \ldots \ldots \ldots \ldots \ldots \ldots$ 


\section{INTRODUCTION}

\subsection{Motivation}

Users of virtual reality (VR) applications are often given the affordance to interact with virtual objects through various selection and manipulation techniques such as ray casting, world in miniature, and virtual hands. However, these interactions are usually entirely virtual and lack haptic feedback. When haptic feedback is provided, it is often limited to forces such as small vibrations provided through a gamepad or hand controllers. Ideally, a user would be able to physically interact with virtual objects through direct hand manipulation. One way to achieve this is through passive haptics, which is the use of realworld physical objects that correspond to virtual objects [1]. Passive haptics can provide a user with additional feedback through physical touch. However, unless a physical space is intentionally built to match a specific virtual reality experience, the ability to perfectly match the physical and virtual objects is limited. To successfully provide users with additional tactile feedback for multiple virtual objects, there is a need to reuse physical props by remapping them to different virtual objects.

Reusing a single haptic prop for multiple virtual objects presents several challenges. First, although physical props can enable a higher sense of immersion and realism during virtual interactions, virtual objects can be represented in any form or size which makes it difficult to provide an accurate physical prop for all cases. However, studies have found that a perfect physical representation of a virtual object used for passive haptics is not necessary to increase the sense of presence and realism [1]. A major challenge that rises with the reuse of physical props is dealing with varying locations of virtual objects. This discrepancy of positions between physical and virtual objects results in a mismatch causing a user to miss or inaccurately touch the physical prop when reaching for the virtual 
representation. Although research has been done using robotics to enable a physical prop to move around depending on specified virtual locations, this method requires expensive equipment and is impractical for most VR users [2]. Without moving things in the physical world, changes must be made in the virtual space to remap the physical prop to an offset virtual object.

Many researchers have engaged in studies involving warping and manipulation of virtual spaces. Through change blindness techniques or subtle changes in rotation of the virtual environment, users can be redirected to walk around within a confined space giving the illusion they are traveling in a larger physical space [3][4]. This idea of virtual manipulation can be used on a virtual hand or limb redirecting a user to reach for an intended target. It has been shown that, to a certain degree, visual sense can dominate the proprioceptive sense in scenarios where there may be a mismatch allowing for some virtual body warping to occur unnoticed [5].

\subsection{Objective}

Our objective is to study multiple techniques for remapping physical reach to guide users towards physical props when reaching for virtual objects and evaluate them in terms of task efficiency and comfort for users. Although virtual warping can technically be applied to all cases of physical and virtual object mismatch, there will be cases where the offset is so great that the techniques are no longer practical for its initial intended use of enabling more realistic interaction. A separate issue to consider is the location of the haptic prop in relation to the user. Comfort is probably the most important factor to consider. People usually do not want to work more than they need to accomplish a task.

The following research questions will be addressed to fulfill the research objective:

- What techniques can remap physical props to multiple virtual objects for reach and direct hand manipulation, and how do these techniques compare in terms of task 
efficiency, comfort, and overall usability?

- To what extent do different distances from the user to the haptic prop and distances between physical and virtual objects influence reaching accuracy, believability, and comfort for hand interactions?

- How can these techniques be applied within an application or game environment?

To address these research questions, we will design, implement, and evaluate two remapping techniques for passive haptics. These techniques will be compared through user studies to gather empirical data on their advantages and disadvantages, as well as inform the comfortable range of offsets and physical prop locations that can be used VR applications. Using the study results, we will then conduct a case study on how to apply the remapping techniques within a game environment as a proof of concept to employing the techniques within applications. 


\section{LITERATURE REVIEW}

The thesis focuses on combining two areas of research which include the use of passive haptics to provide additional tactile feedback and manipulation of virtual hand position and movements.

\subsection{Haptics in Virtual Reality}

Haptics has a long history of use in many industries including aerospace, medical, and entertainment [6]. For virtual reality, it is used to provide additional feedback to users as most applications for VR only focus on visual and auditory feedback. There are two general categories of haptics: passive haptics and active haptics. Passive haptics is a category of haptics where objects or devices provide tactile feedback naturally through a users sense of touch of their physical properties. In contrast, active haptics, generate haptics through use of software and actuators on physical devices.

Haptic devices are still being researched to provide a more active way of providing force feedback as opposed to passive haptics which are still situated props or objects within the physical space that the user interacts with. Haptics is an area where robotics plays a role as they can essentially be physical props that can move. McNeely discusses the concept of robotic graphics that uses robots to enable force feedback for virtual reality [7]. McNeely discusses the idea of robotic shape displays (RSD) which utilize robots that are specifically used to provide tactile feedback by repositioning and reorienting themselves based on anticipated movements of the VR user. He et al. [2] also uses robots to provide haptic feedback in research regarding the use of haptic proxies to enable collaborative tasks in VR. The robot moves around on a table in the physical world to accommodate changes done by the other user that is collaborating on the task.

Although robots and other active haptic devices have the benefit of dynamically adapt- 
ing to match changes in a virtual environment, these methods are costly and not practical for most users. Passive haptics have the advantage of being easily accessible as they can be any house hold item such as a bottle, cup, or box. In his research, Insko [1] provided evidence that passive haptics can enhance a users virtual experience in various ways including spatial knowledge, cognitive mapping, and overall presence within the virtual environment. Passive haptics can provide users with more control and allow for better correlation between virtual and real world tasks such as handwriting in virtual reality as researched by Poupyrev et al. [8].

\subsection{Visual and Proprioceptive Mismatch}

There has been evidence to show that visual feedback can dominate proprioceptive senses when there is a mismatch between the virtual and physical world [5]. Many researchers have taken advantage of this to provide virtual experiences that would not be possible within the physical world the user is situated in. For example, Razzaque et al. [4] studied the use of slight rotational adjustments in the head to enable redirected walking and create the illusion that the user is in a larger space.

There have been studies regarding the effects of mismatch in a closed-loop physical reaching task. Ebrahim et al. [9] found that although tactile feedback can provide more accurate depth judgment in physical reaching tasks, the performance can be significantly altered when visual and proprioceptive information do not match. Although this research provides evidence of change in task performance the study focused mainly within a small scope of a single reaching task. Effects and task performance can also be affected by location of a target which will be explored in this research.

Poupyrev et al. [10] proposed the Go-Go technique which allowed users to interact with distant objects through amplified hand movements which closely relates to the interpolation technique in our research when virtual objects are placed farther away than 
the physical object. Poupyrev et al. [11] goes on to compare the Go-Go technique to a ray-casting selection and manipulation method and found it to be more accurate in object selection tasks and comparable for object positioning.

Understanding how people can adapt to these changes overtime is also important. In addition to finding the effects of the presence of an arm or full body avatar in task performance, Bodenheimer et al. [12] found that people adapted overtime to a lateral displacement of the visual environment through a throwing task. Kohli et al. [13] found that although there are indications of reduced performance in warped virtual spaces, after adaptation, users can perform tasks when their visual senses do not fully match their proprioceptive sense about equally to a one-to-one virtual environment. For this research, we also will study the affects of the remapping techniques on task performance over time, but in contrast to previous studies we will look at the effects over a larger set of tasks and offsets with two different techniques.

\subsection{Redirecting Reach}

Similar to the research proposed in this thesis, there has been prior research involving the combination of manipulation of virtual movements to provide redirected movements towards an intended physical object. In research by Kohli et al. [14], a concept of redirected touching was explored where virtual space was warped to provide haptic feedback for multiple virtual objects with a single physical object. Kohli's research goes on to understand the effects of the warping on task performance in a target touching task where a physical board is placed in front of participants [15].

In recent work, Azmandian et al. [16] studies the use of haptic retargeting where different techniques are used to remap a hand to reach and grab a physical object. The research discusses the use of body warping, world warping, and a hybrid of both. Body warping manipulated the virtual arm and hand to offset as it reaches towards a virtual 
object where as world warping remaps a virtual object by rotating the virtual world around the user's head which is applied as the user looks away from the object. Further, Cheng et al. [17] discusses applications of hand redirection through use of Sparse Haptic Proxies which are defined as a set of geometric primitives that provide touch feedback for various objects in a virtual environment. The study explores the use of the Sparse Haptic Proxy in two vastly different environments to show how it can be used to provide tactile feedback for a variety of scenarios. Although there has been research done involving remapping of reach in virtual reality, there is still a need for evaluation of other techniques and how these can be applied to a seated experience with a single prop. When seated at a table, there are several factors to consider including the distance and location of the object as well as the direction of offset between physical and virtual objects. All of these factors will be explored in this thesis and how the techniques can be applied within an interactive game. 


\section{RESEARCH OVERVIEW}

The research aims to answer the questions stated in the introduction of what techniques are available to remap physical props to virtual objects and how do they compare for use in a reaching task. We also want to understand at what point do users start to notice or feel uncomfortable with the remapping techniques. Once this information is gathered we will implement the technique with changes based on the data to optimize comfort for the user within a game environment to show a possibility of how it may be applied within an application.

To cover all questions, the research plan includes two user studies and a case study. The first user study will focus on the comparison of two virtual hand remapping techniques: translational shift and interpolated reach. The second study specifically aims to understand how people adjust to manipulation of virtual hand positions and movements. Finally, the case study will be used to apply what was learned from the previous studies as a proof of concept where participants will be immersed within a virtual game environment while playing a basic game requiring the use of the remapping techniques.

\subsection{Technique Design}

Our research focuses on the use of a single haptic prop to map to multiple virtual objects. Therefore, all three studies will involve the use of a single tracked object where participants will perform tasks while seated at a desk interacting with virtual objects that are of similar size and shape. The two techniques that will be evaluated are translational shift and interpolated reach.

1. Translational Shift involves repositioning the virtual hand at a new location based on the difference in position between the physical and virtual objects. This results 
in a virtual hand that still moves one-to-one with the physical hand, but is visually offset from its original location.

2. Interpolated Reach is a technique that aims to keep the virtual hand at its original location, but instead adjusts the speed and trajectory of the virtual hand as it moves toward the virtual object to compensate for the offset between the physical and virtual objects. The hand starts at no offset at its starting position to a full offset when it reaches the object.

One other technique for remapping passive haptics involving virtual world rotations around a user's head as done in the study by Azmandian et al. [16] was considered, but we opted to work with techniques involving only virtual hand manipulation. In addition, the world warping technique alone is not applicable to all virtual and physical offset positions as they need to be aligned within the path of rotation.

\subsection{Experiment 1}

The first study focuses on understanding the effects of the techniques on a simple object grasping and placing task. Both techniques were presented to the participant along with a one-to-one mapped version used as a baseline for comparison. Each set of tasks will involve a different combination of physical object position and offset distance. The data collected includes the time taken to reach the physical object, time taken to place on a target, any errors that may have occurred including bumping the bottle or missing it entirely, and a recording of hand movements. A semi-structured interview was conducted at the end of the study to collect additional feedback from the participant and discuss how they performed during each set of tasks, what changes they noticed, and what preferences they had between the techniques. 


\subsection{Experiment 2}

Experiment 1 used a different technique with a different configuration of prop positions, techniques, and distances between physical and virtual objects, so it may have been difficult for participants to adapt to the constantly changing techniques. In Experiment 2 , we studied whether a participant can adapt to using the same type of technique with a constant offset in repeated trials. Participants will also switch from the offset version to a one-to-one version after a set number of trials to understand how the techniques affect readaptation to one-to-one tasks.

\subsection{Case Study}

A final case study was done to understand how the remapping techniques can be applied within an application with multiple virtual objects. To do this, we designed and implemented a game involved moving multiple virtual objects to various tables using only a single physical prop. The task requires frequent remapping to different objects. Using this application, we collected feedback about usability and preferences about the different techniques through informal user testing. 


\section{TECHNIQUE IMPLEMENTATION}

\subsection{Materials}

The techniques were tested with a task that involved reaching for an object (a plastic bottle) on a table. All participants sat in a stationary (non-rotating) chair at a $72 \times 30$ inch table. The studies used an Oculus Rift CV1 head-mounted display (HMD). An Oculus Rift remote was used by the experimenter to trigger tasks and log any errors that occurred such as missing or bumping the bottle. The study software applications were developed in the Unity engine and ran on a computer running 64-bit Windows 10, a $3.4 \mathrm{GHz}$ Quad Core processor, and a GeForce GTX 1070 graphics processing unit.

Six degree-of-freedom (DOF) motion tracking was handled using an OptiTrack capture system with 12 Flex 13 cameras. Based on manufacturer reports, each camera recorded tracking data at a frame rate of 120 frames per second and operated with 8.33 ms latency. Six DOF head-tracked viewing was enabled for the HMD, and hand tracking was enabled by attaching a rigid body to the participant's right wrist via a Velcro strap. A rigid body attached to an elastic band was also worn on the tip of the middle and ring fingers, which was used to control the grasping action and animation of the virtual hand. With this configuration, the thumb was not tracked, and the other four fingers moved together to open or close during grasping motions.

The studies used a cylindrical plastic bottle as a passive haptic prop, which was tracked by a rigid body. Soft fabric was added to the bottom of the bottle to reduce noise when the experimenter placed it on the table. Noise reduction was important for this experiment in order to eliminate any sound queues as the experimenter was required to move the bottle to different positions on the table during the experiments. Participants used only their right hands for interactions in all experiments. Figure 4.1 shows the physical setup. 

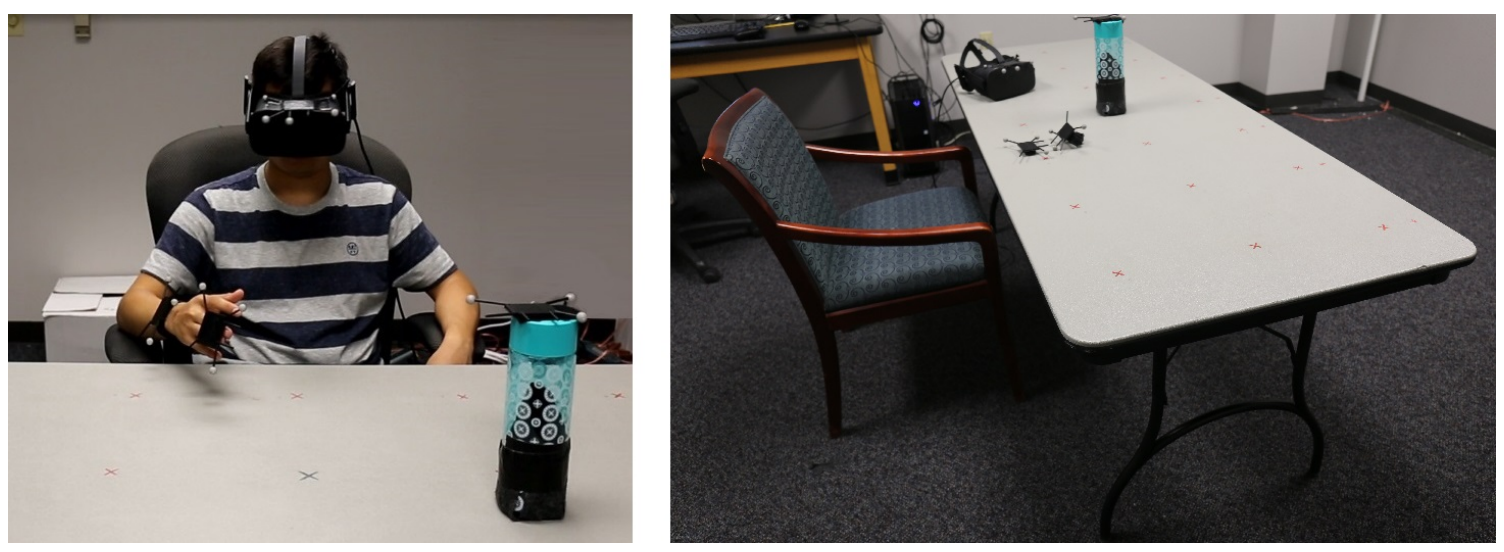

Figure 4.1: The physical setup used for the experiments includes a table, a stationary chair, a tracked bottle, and hand markers, and an HMD.

\subsection{Translational Shift}

Translational shift is a remapping technique that involves relocating the virtual hand based on the positional offset between the real world object and the virtual object. The position of the tracked real-world object is constantly tracked, so a new offset can be calculated for a new virtual object position at any given time. Figure ??, left, demonstrates the basic concept using semi-transparent blue objects to show where the positions of tracked objects would appear with a one-to-one mapping. The calculation for the virtual hand position, $P_{v h}$, using translation offset is given by:

$$
P_{v h}=P_{p h}+\left(P_{p o}-P_{v o}\right)
$$

Here, $P_{p h}$ is the physical hand's current world position and $P_{p o}$ and $P_{v o}$ are the world positions of the physical object and virtual object respectively. Figure 4.2 demonstrates the technique. Translational offset can be applied in any axis depending on the offset. In the case of our studies, we focused on offsets only in the horizontal plane. 


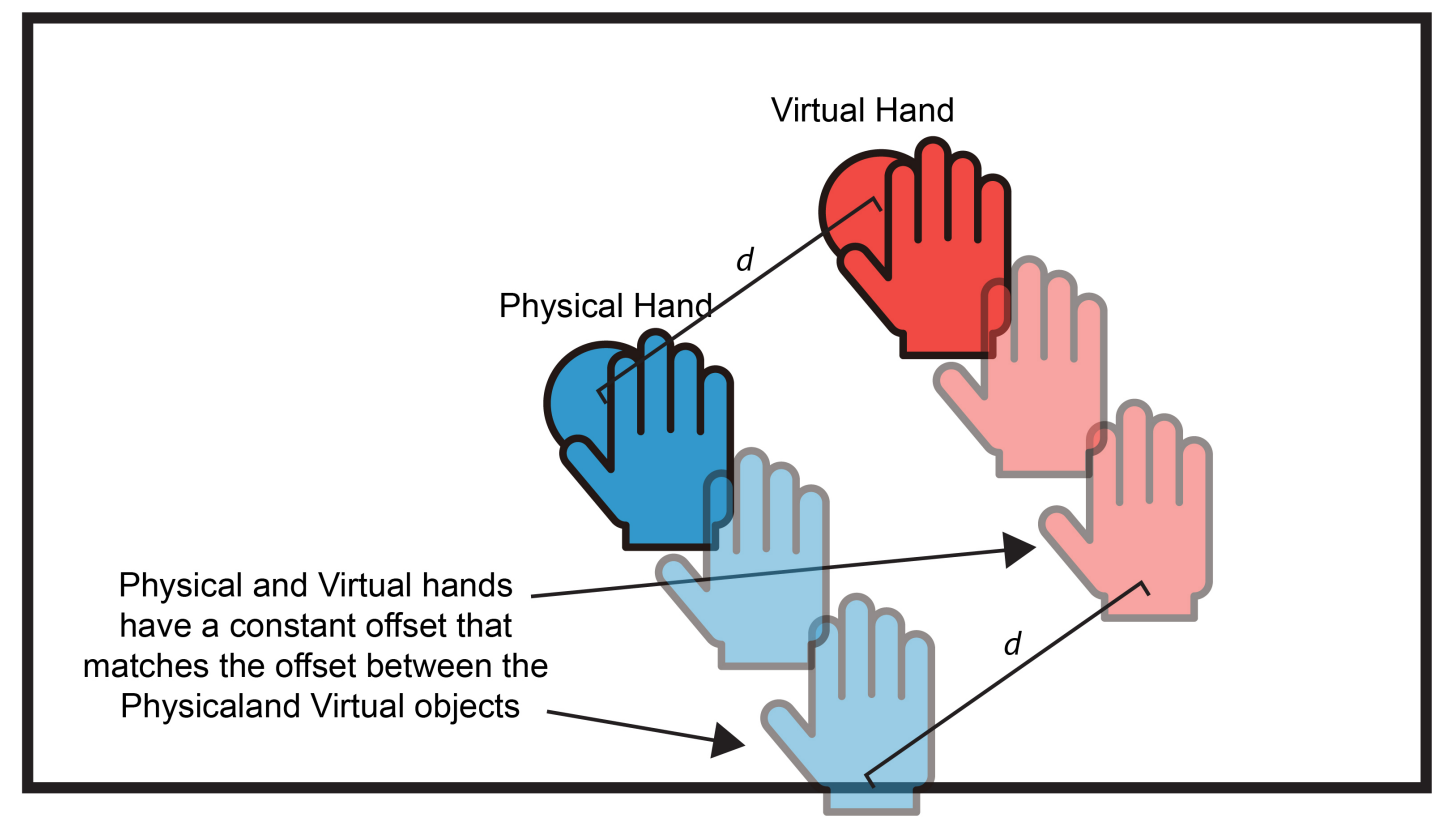

Figure 4.2: A top-down diagram demonstrating translational shift. This shows the physical hand and object in blue and the virtual hand and object in red. Here the physical and virtual hands maintain a constant offset based on the distance between the physical and virtual objects which is denoted here by $d$.

\subsection{Interpolated Reach}

Interpolated reach is a technique that aims to initially keep the virtual hand at a starting position that matches the physical real world hand so the virtual hand appears to be where it would be in relation to the user's body. As the physical hand moves, an offset is gradually applied to the virtual hand as it moves toward the virtual object until it reaches the maximum offset at the time the physical hand reaches the physical object. It is expected that the user will gradually adjust his hand position during reaching so the virtual hand will reach the virtual target, and this will guide the physical hand to the physical prop at the same time. The technique uses the calculated offset between the virtual and 


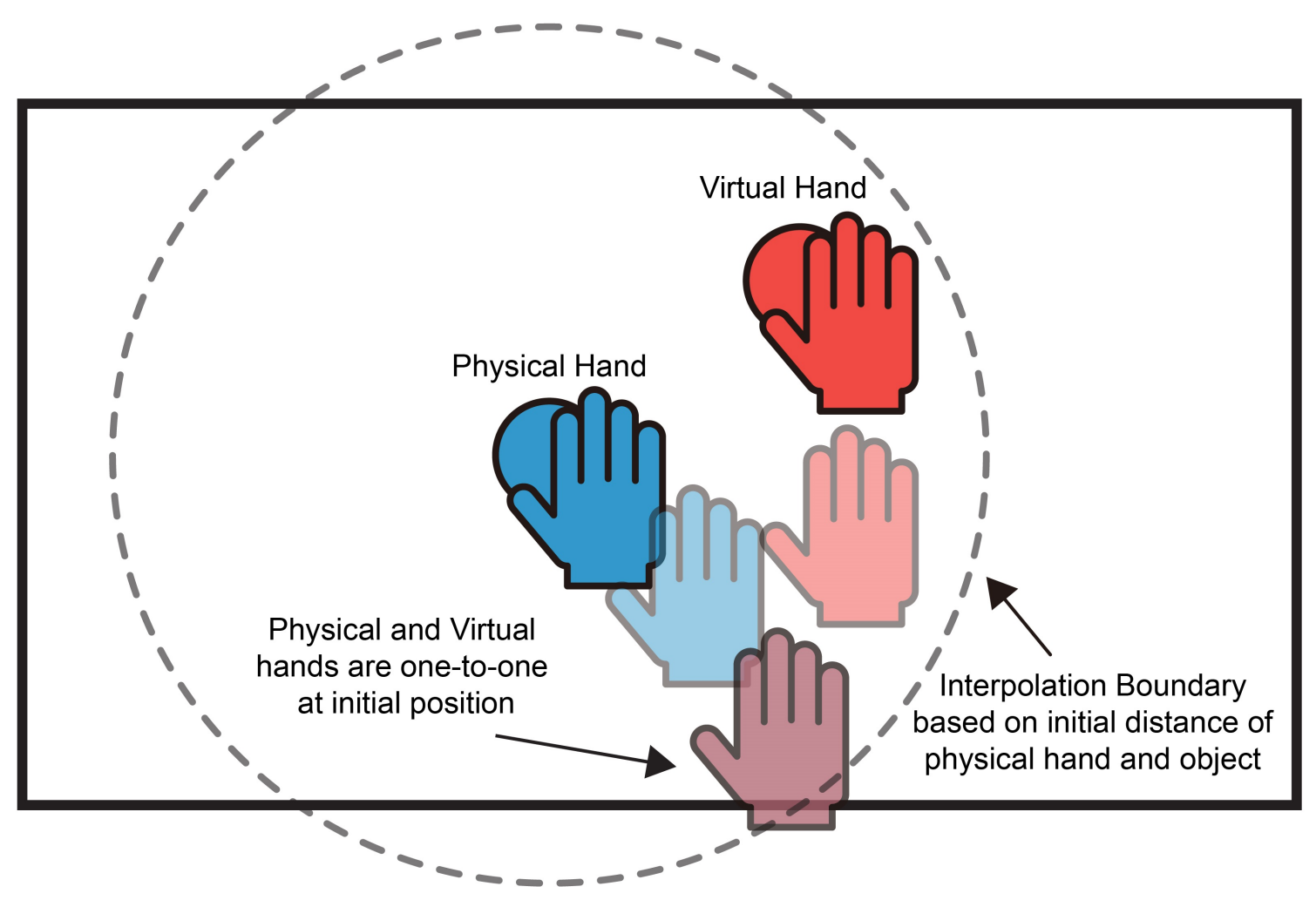

Figure 4.3: A top-down diagram demonstrating interpolated reach. This shows the physical hand and object in blue and the virtual hand and object in red. The dotted circle has a radius equal to the initial distance of the physical hand and object, and it denotes the region where interpolation is in effect. When the physical hand is outside this boundary, a one-to-one mapping is used.

physical object to dynamically blend between the physical real world hand position to its offset position. Because of this dynamic changing of hand position, the virtual hand experiences manipulations in its speed and trajectory during reaching tasks to compensate for the offset. Figure 4.3 demonstrates the concept.

The virtual hand position, $P_{v h}$, for interpolation is given by the following equation:

$$
P_{v h}= \begin{cases}P_{p h}, & D \geq B \\ P_{p h}+\left(P_{p o}-P_{v o}\right) *\left(1-\frac{D}{B}\right), & D<B\end{cases}
$$


Here, $P_{p h}$ is again the physical hand's current world position, and $P_{p o}$ and $P_{v o}$ are the world positions of the physical object and virtual object, respectively. We have an additional term, $\left(1-\frac{D}{B}\right)$, which is the offset control value that determines how much of the offset is applied to the virtual hand. Here $D$ is the distance between the physical object and physical hand. As the hand moves closer to the real world object, $D$ gets closer to 0 . The term $B$ is the interpolation boundary, which is a constant value that determines the region where interpolation is in effect, as given by:

$$
B=\left|P_{p o}-P_{p h}\right|+0.1
$$

In this implementation, the boundary offset is given by the initial distance between the physical hand and physical object plus a small buffer value of 0.1 meters. The buffer value added to the boundary offset is needed to ensure the hand stays within the interpolation region regardless of initial hand movement. Without the buffer, the hand starts at the very edge of the boundary region, and there is potential for the user to reach out in a direction that moves away from area of effect causing them to miss the object completely as the offset is not being applied. When $D \geq B$, the hand is outside the region of effect, so the virtual hand reverts to a one-to-one mapping with the physical hand. 


\section{EXPERIMENT 1}

\subsection{Goals and Hypotheses}

Although past research has shown that remapping reach techniques can be applied to reuse physical objects for providing tactile feedback of multiple virtual objects, there is still the question of how these techniques affect a user's ability to perform tasks when compared to one-to-one mapping of the virtual and physical hand. Specifically, our research aimed to compare two remapping techniques for seated VR experiences. It is important to understand the limitations of these techniques in order to use them effectively and comfortably within applications. The goal of the first experiment was to test various conditions of the two remapping techniques to determine what combinations and ranges of offsets and table positions for the physical prop are best in terms of task performance and comfort.

We hypothesized that between translational offset and interpolation, translational offset would perform better overall as it still maintains one-to-one motion in contrast to the changing of speed and trajectory of the hand in interpolated mapping. Logically, we believed larger offsets and and physical prop positions that were farther away from the user would cause decrease in task performance.

\subsection{Experimental Task and Environment}

The study was conducted in a motion capture space tracked with OptiTrack cameras. Participants were seated in a non-rolling chair with arm rests at the rectangular table. The table was marked to determine physical positions for the physical prop. The marks were placed 10 inches apart from each other starting from the center of the table. The chair and table positions were marked to ensure that each participant was using the same configuration for the physical setup.

To make sure participants were focused on the task, the virtual environment was made 


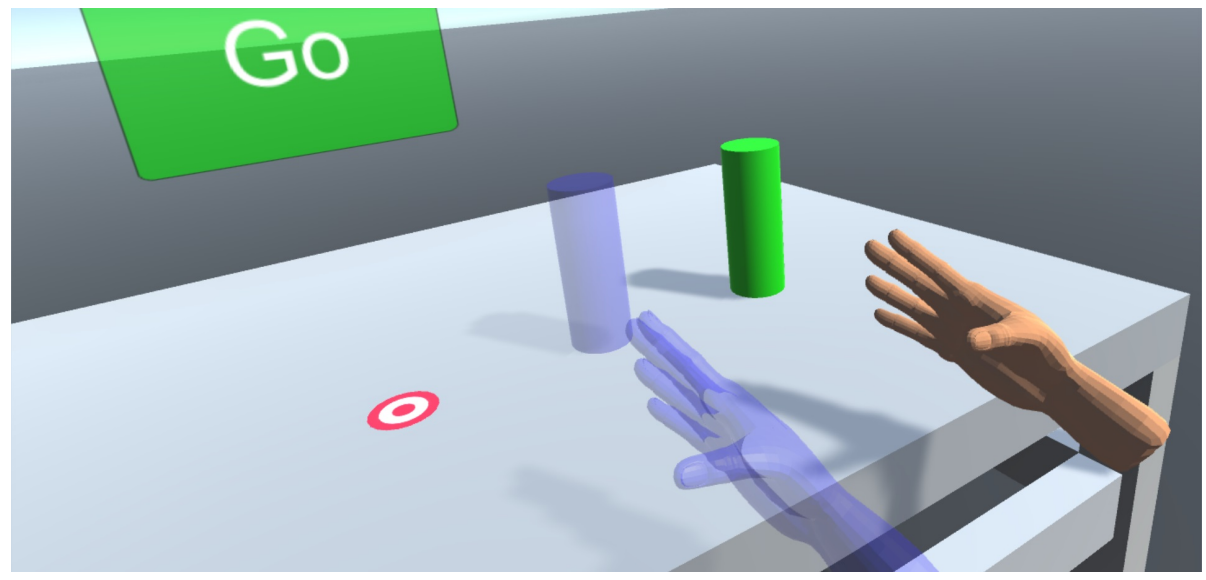

Figure 5.1: An image of the experimental environments shows how a green "GO" sign was presented to the participant to signal them to reach the green cylindrical virtual object and place it on the red target. Here the physical hand and object are represented in blue. The physical components would not be visible to the participant and are shown only to demonstrate the effects of the hand remapping.

simple with an open environment with only a white rectangular table placed in front of them that matched the size and shape of the real world table. The only other other objects in the environment consisted of a gray rectangular sign that was placed at eye level behind the table, the cylindrical virtual object that they interact with, and a red target.

The experimental task was to use the virtual hand to reach and grab the virtual object after its color changed from red to green and the overhead sign changed to a "GO!" label. Directly after grabbing the object, the participant placed the object on a red target that was also placed within the table area. This task was triggered with a remote controlled by the experimenter. Time was calculated between start and grasp and from grasp to target placement. The grasping of the object was recorded through the experimenter's observation and confirmed by clicking the button on the Oculus Remote. 


\subsection{Experimental Design}

In the first experiment, we studied how reach time and accuracy can be affected by various conditions in an object reaching task. For mapping virtual to physical objects, there are various conditions that can change the overall difficulty of the task. For this experiment we compared the following conditions:

- Technique: As described in our research overview, we looked at two remapping techniques: translational offset and interpolation. Participants were also given tasks using a direct one-to-one mapping of the real world hand to the virtual hand as a basis for comparison.

- Offset Size: Offset size is the distance measured between the physical and virtual object. We studied a total of 4 variations of offset size: 10, 14, 20, and 30 inches. The 14-inch offsets were exclusively used for diagonal offsets, as each marker on the table was placed 10 inches apart from any adjacent marker, making the diagonal distance approximately 14.14 inches.

- Offset Direction: Along with the 2D object distribution of table locations, it was possible to have different 2D directions for the offset vector between the real and virtual objects. We find it most helpful to consider consider two cases of offset direction, which we call toward and away. An offset is considered toward a user if the virtual object appears closer to the user than the actual physical object (in other words, the virtual object is between the physical object and the user's physical hand). An offset is considered away when the virtual object appears farther than the physical object from the user's point of view (in other words, the physical object is between the virtual object and the user's physical hand).

- Table Position: We use the term table position to refer to the location on the table 
where the physical object is. The experiment used 17 possible locations on the table. Figure 5.2 shows the approximate configuration of locations by square cells. Each location was separated from others by 10 inches to the left and right, and by 10 inches in the direction away from the user. During the experiment, the experimenter adjusted object placement at table positions by manually moving the object.

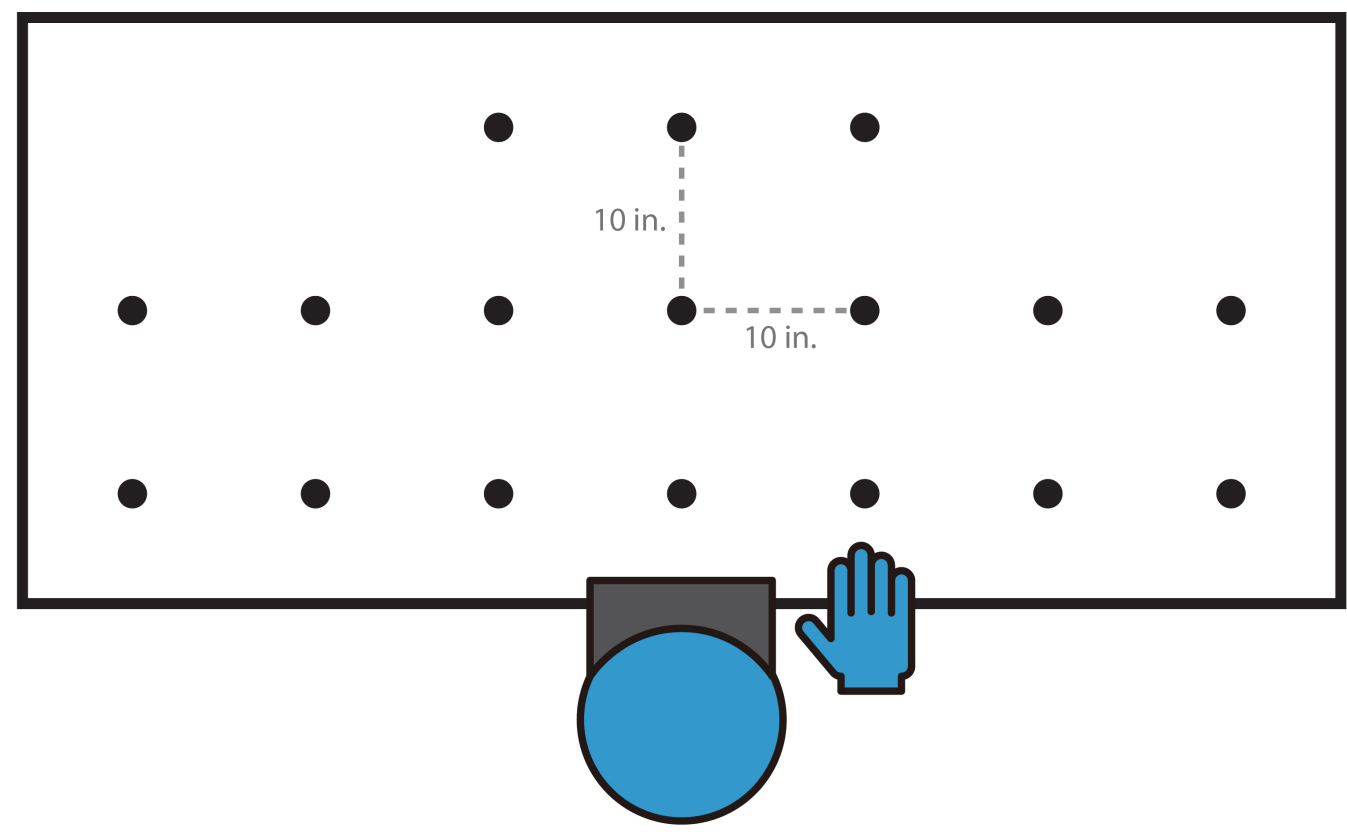

Figure 5.2: A total of 17 table positions were used in Experiment 1 marked in this image as black dots. Positions were marked on the table and placed separated by 10 inches from other dots along the table axes. The top right and top left corners were unused as the distances were out of the comfortable reaching range.

The experiment was conducted with a repeated-measures design. In other words, each participant was exposed to all possible task variations during the experiment. The experimental procedure was broken up into three parts, each using a different hand mapping 
technique: normal one-to-one reach, translational shift, and interpolated reach. All participants started with the one-to-one version for the first block of trials. The one-to-one block consisted of 17 tasks which covered each possible marked location on the table. For the translational shift and interpolated reach blocks, participants were asked to perform a set of different tasks based on unique variations of the independent variables. Because of the numerous possible combinations of the independent variables, a subset of 42 tasks for each technique was chosen for the experiment. Each of the 42 tasks were unique with a different combination of offset size, offset direction, and object position. The tasks were derived to cover all possible table locations with offset directions alternating between every other location. To ensure the virtual object always appeared as it was resting on top of the table, the virtual object was never offset beyond the virtual table borders.

Although each participant was given the one-to-one session to begin with, the other two sessions with the remapping techniques were counter balanced so that half of the participants started with translational shift and the other half started with interpolated technique. Task ordering within each technique block (including the one-to-one session) was randomized for each participant.

To measure the effects of the independent variables on reach performance, we considered time and errors as dependent variables. Reach time was measured as the time from when the task started, signified by the "Go" sign and the virtual bottle changing from red to green, to the end of the task, when the participants successfully grasped the object and all five fingers made contact with the physical object. Times were recorded by the experimenter where both the start and end of the reach task were manually marked through clicking a button. Because people might normally reach at different speeds, and different table positions will have different times due to different distances, we analyzed reach time for each condition by taking the time difference between the measured reach time and the participant's corresponding time for the baseline reaches with one-to-one movement for 
each given table position.

To measure reach errors, we considered two types of errors: bumping and missing the physical object. A bump error was recorded if the hand made contact with the bottle, including knocking it over, without a successful grasp. A miss error was recorded if the hand reached past the physical object without a successful grasp. Errors were manually noted by the experimenter observing the participant. The error metric uses the total number of all errors (of either type) observed per trial.

\subsection{Procedure}

At the beginning of the experiment, participants were seated at a table and given an overview of the study and equipment used including the tracking system and Oculus Rift. They were then required to sign an informed consent form before proceeding with the experiment. Each participant filled out a brief background questionnaire that included questions regarding demographic information (age, gender, and occupation) and their overall experience with 3D games and virtual reality.

The participants were then assisted in putting on the HMD and markers to track the hand. Before beginning the main study, a practice session was provided to the participant. The purpose of the practice session was to get the participant accustomed to the task and virtual environment before the main experiment. The practice session included five oneto-one reaching tasks so participants were not introduced to any of the two remapping techniques beforehand. After the practice session, participants were asked to remove the HMD for a short break while measurements were recorded for their height and arm length.

The main study started once the short break and measurements were complete. The main study consisted of three sessions with a mandatory five minute break between each. Each session included a set of tasks presented in random order. The task involved reaching a virtual cylinder and placing it onto a target on the table. Before each task the virtual hand 
and object are hidden from view. When the task begins, the virtual hand, object, and target fade in to view and the word 'READY' is displayed on a gray instructions panel. At this time the virtual object is red. During this time, the participant was asked to place their hands on the arm rest and not move and simply acknowledge the location of the virtual objects. After approximately 2 seconds, the experimenter initiates the task by clicking a remote button which changes the gray 'READY' sign to a green 'GO' sign and changes the virtual object from red to green. This signals the participant to reach the object and place it onto the red target. Time is recorded between the appearance of the 'GO' sign and the object being placed onto the target. Once the object is placed on the target, the hand, target, and virtual object fade away until the next task is started. Participants were asked to perform the tasks as quickly as they could in a way that was comfortable and natural to them to avoid error. Errors that involved the participant missing or bumping into the bottle were recorded by a remote click manually from observation by the experimenter.

The study consisted of three sessions in total with a mandatory 5 minute break between each. For the first session, all participants began with the one-to-one mapped version that consisted of 17 reaching tasks that covered each marked table position. The second and third sessions consisted of 42 reaching tasks each using either translational offset or interpolation. The technique order was alternated between each participant. Half of the participants did translational offset tasks for session two while the other half had interpolation.

After the three sessions, a semi-structured interview was conducted by the experimenter to collect additional feedback from the participant. The interview consisted of questions regarding how they felt about the different versions and tasks they experienced and what differences they noticed. Experiment 1 took approximately 1 hour. 


\subsection{Participants}

Sixteen university students ( 8 male, 8 female) took part in the Experiment 1 . The participants' ages ranged from 20 to 29 with a median of 25 years. Ten of the 16 students

were in the Visualization program while the rest were computer science or engineering majors. Participation was voluntary and no compensation was provided. Nine participants reported spending at least one hour a week playing 3D video games and 11 reported having prior experience with virtual reality. All participants were required to use their right hand for the experiment. Handedness or hand dominance was not surveyed as all participants' reach times were measured in reference to their own one-to-one reach times.

\subsection{Results and Discussion}

Experiment 1 primarily tested two different reach techniques with different offset sizes. While the experiment also considers many different table positions and offset directions, we summarize these differences based on toward and away offset directions and by close and far table positions. Offset direction was based on the direction vector from the physical object to the virtual object relative to the user's seated position. If the virtual object was at a farther position than the physical object from the user it was considered an away offset direction and conversely if the virtual object ended up closer to the user than the physical object, the offset direction was considered toward. Figure 5.4 illustrates some examples of how these offset directions were determined. The object distance were labeled either far or close based on the table position's distance to the user's right hand. Figure 5.3 illustrates how the table positions were categorized. 


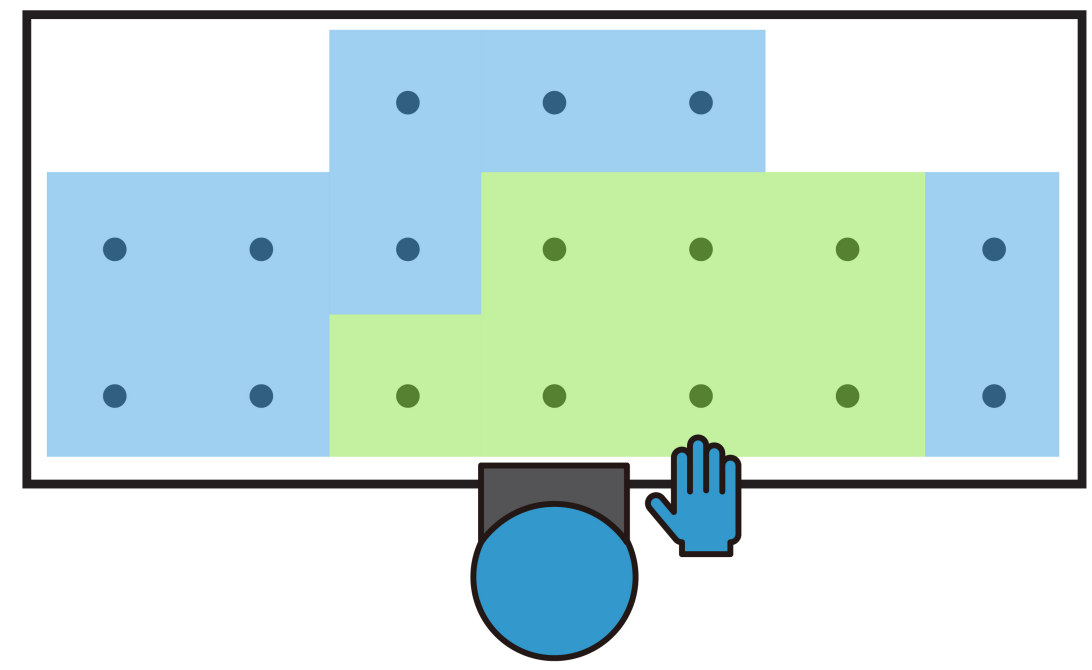

Figure 5.3: Regions for Object Distance. Objects placed at table positions highlighted in green were counted as a "close" reach target while table positions highlighted in blue were counted as a "far" reach target.

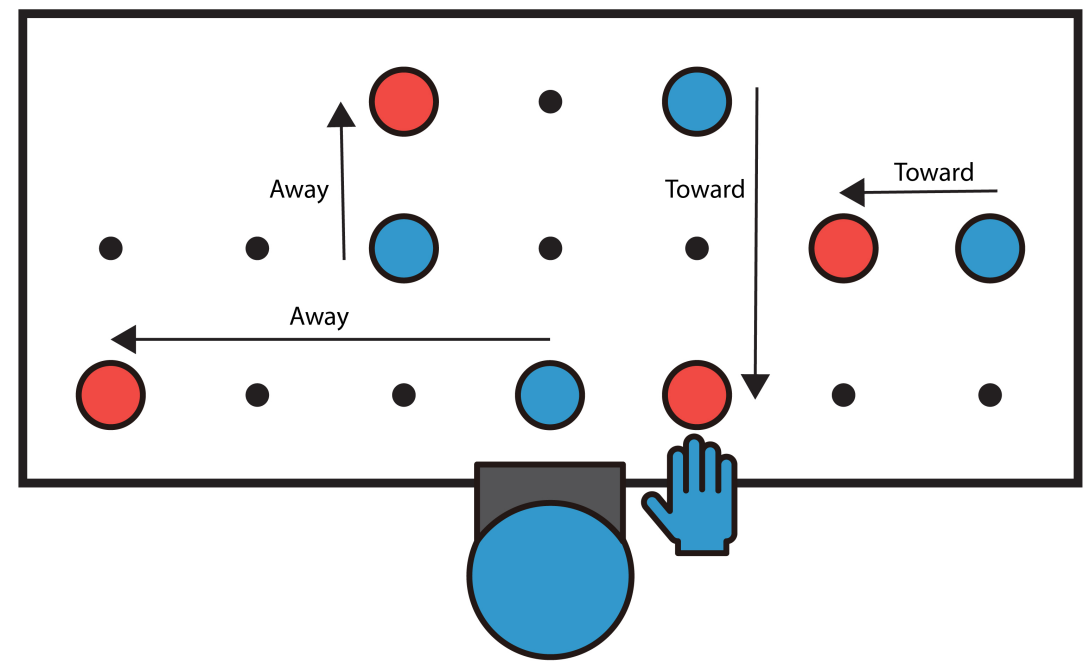

Figure 5.4: Examples of offset directions. Here a blue circle represents the physical object and a red circle represents the virtual object. 


\subsubsection{Quantitative Results}

Because of the number of factors involved in experiment 1 , it would be difficult to comprehend an analysis of all possible combinations. Instead, the quantitative results are presented based on different relevant subsets of factors. For our analysis and presentation of results, we use repeated-measures ANOVA tests and graphical plots to represent differences due to experimental variables. The following reported results cover only the test statistics for significant effects. The results are presented using a box-and-whiskers plots where the colored rectangles represent the interquartile range (IQR) and the horizontal black lines within the rectangles mark the median value. The vertical black "whisker" lines extend from the rectangle to the most extreme value that falls an additional half-IQR beyond the IQR, or no whisker is shown if no points fall in this range. Black dots in the plot represent values beyond this range.

For Experiment 1, the time results met the assumptions of normality and sphericity for parametric testing. Error results sometimes met the assumptions for parametric testing, however some error subsets were skewed right due to most trials have few and usually no errors. We were unable to correct the distributions with data transformations or to identify non-parametric alternatives for our sample sizes. For this reason, and considering the robust nature of ANOVA tests for normality violations (e.g., [18]), we chose to rely on parametric repeated-measures ANOVAs with the acknowledgement of increased risk of type 1 errors (e.g., [19]).

In our tests, we consider performance for the two different techniques, different offset sizes, and offset direction (the virtual object offset being away from user or toward the user). We ran three-way repeated-measures ANOVAs for reach time. Reach time is calculated based on the difference between the time to complete a task and the baseline one-to-one time that was measured during the one-to-one session for that particular table 
position. The distribution of times are shown in Figure 5.5. The results show that the interpolated reach technique was significantly slower overall than the translational shift technique with a significant main effect yielding $F(1,15)=4.83$ and $p=0.048$, but this is better explained by interaction effects (to be explained in the following paragraph). The effect of offset direction was also significant $F(1,15)=148.49$ and $p<0.001$, showing that reach speeds were significantly faster when the virtual object was offset away from the body than when offset toward the body. Additionally, offset direction had a significant main effect with $F(3,45)=27.83$ and $p<0.001$, with posthoc Bonferonni-corrected pairwise comparisons showing offsets of size 30 were significantly slower than all other offset sizes. It is interesting to note the failure to detect significant performance differences with smaller offset sizes. This suggests that people may generally be able to tolerate and compensate for substantial mismatches between virtual and physical objects without major problems when the offset size is less than or equal to 20 inches. Further experimentation with finer-grain offset sizes would be needed to more precisely assess any tolerance threshold.

Further evidence of the relationship among the experimental factors is given by a significant three-way interaction among technique, offset size, and offset direction, with $F(3,45)=5.48$ and $p=0.002$, and by a significant two-way interaction between offset size and direction, with $F(3,45)=10.14$ and $p<0.001$. From Figure 5.5, we can see that slower reach times were observed for towards reaches compared to away reaches, and the problems with the largest offsets were significantly worse with the away movements. Most interestingly, the translational technique resulted in relatively good speeds for away offset directions, and this combination seemed to be most tolerant to large (30 in) offset sizes. On the other hand, the worst combination for speed was the interpolated reach in the toward direction and with 30-in offsets.

We also tested for effects on reach errors due to technique, offset size, and offset direc- 
tion with a three-way repeated-measures ANOVAs. The test found significant main effects for both technique and offset size, but these effects are better explained by a significant interaction between the two factors with $F(3,45)=4.62$ and $p=0.007$. The interaction shows that greater numbers of errors were made in conditions using interpolated reach with higher offset sizes. In contrast, with the translational technique, errors were much more similar among offset sizes (see Figure 5.6).

When looking at the the time and error results together, we can see that the translational shift technique is more robust for better performance with larger mismatches between virtual and physical targets. Also, the interpolated technique did especially poorly for 30-inch offsets in terms of both time and errors. This suggests the existence of a limit to the offset size before seeing a dramatic penalty to performance. Though reaching away from rather than towards the body allowed significantly faster reaching, the ANOVA for errors failed to detect a significant effect of reach direction.

We also considered effects on performance based on table position. By categorizing table positions as either far or close from the right hand's starting position based on distance threshold as seen in Figure 5.3, we were able to conduct statistical testing. A two-way factorial repeated-measures ANOVA found a significant main effect in distance with $F(1,15)=36.33$ and $p<0.001$, showing reach times were affected more by the remapping techniques when an object was farther away. Unsurprisingly, reaching times were longer and more errors were observed for reaches when the prop was positioned far from the default position of the user's right side. A distribution of performance results by table position is summarized in Figure 5.7. This distribution was observed independent of other factors. 


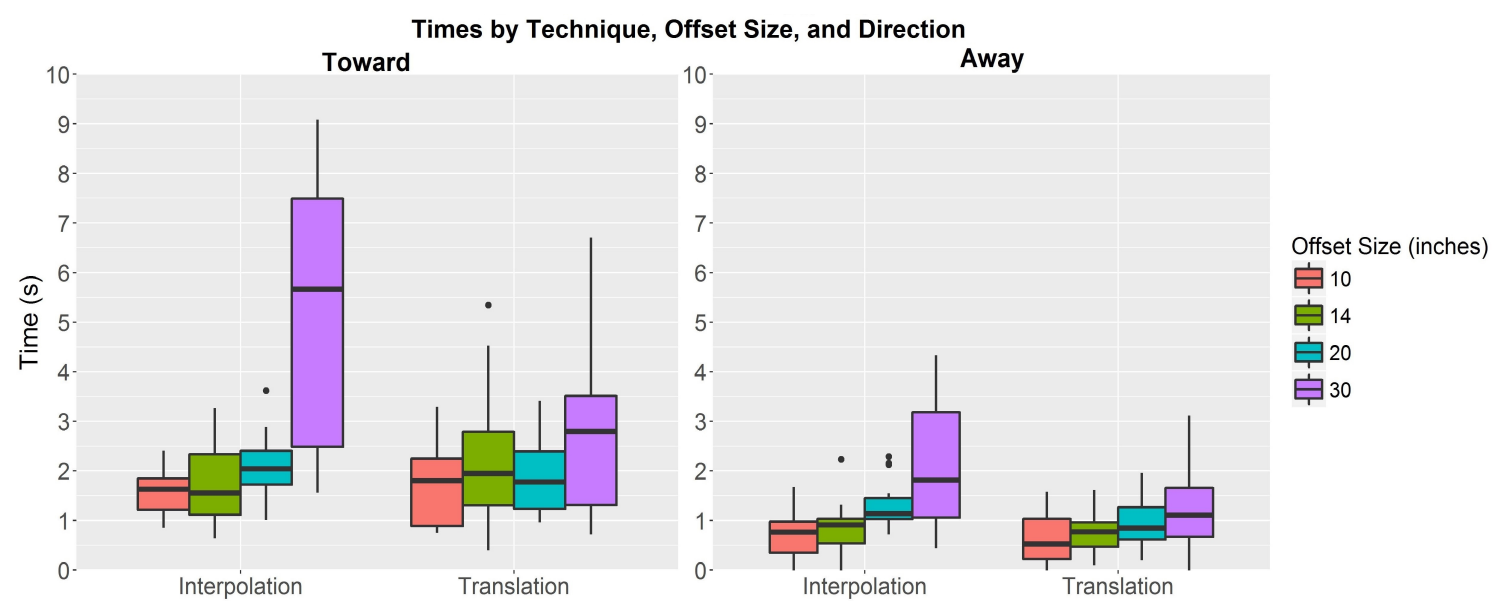

Figure 5.5: Reach times from Experiment 1 separated by technique type, offset direction, and offset size. Speeds are similar for small to moderate offset sizes. The translational techniques are significantly more robust to large offset sizes, and reaching away from the body is significantly faster than reaching towards the body.

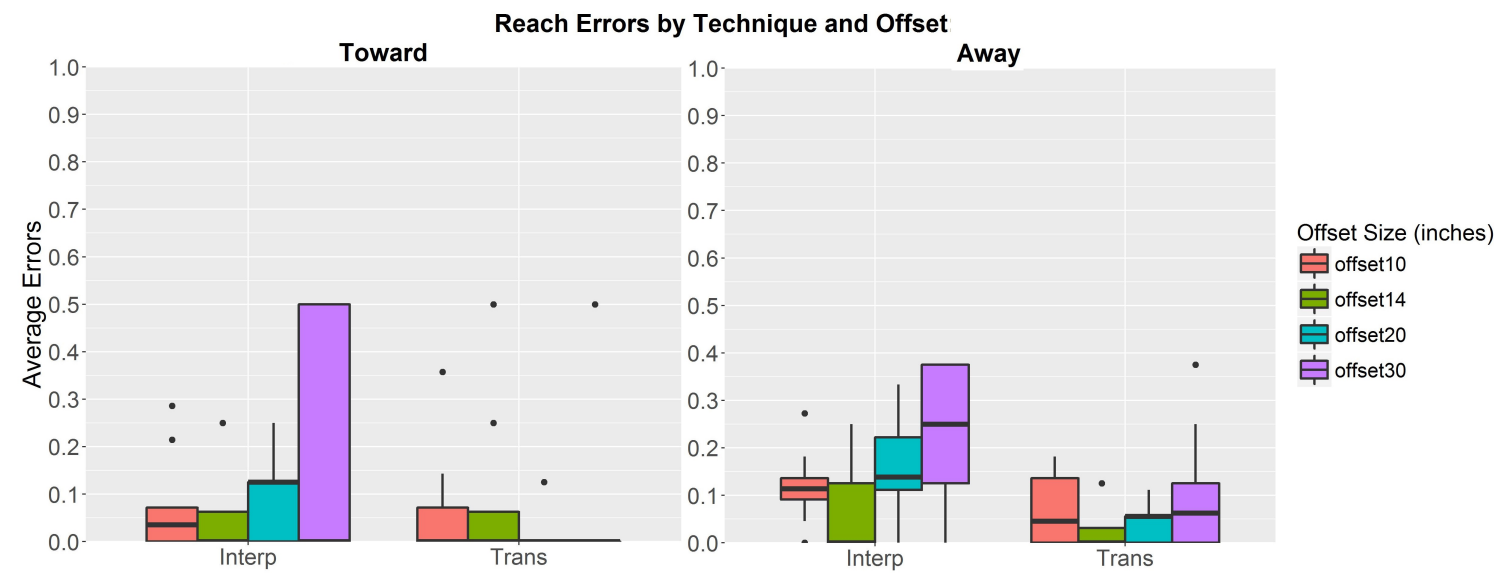

Figure 5.6: Errors from Experiment 1 separated by technique type, offset direction, and offset size. Errors for the reach task in Experiment 1 were significantly worse with the interpolation technique when used with high offset sizes. 
Average Reach Time

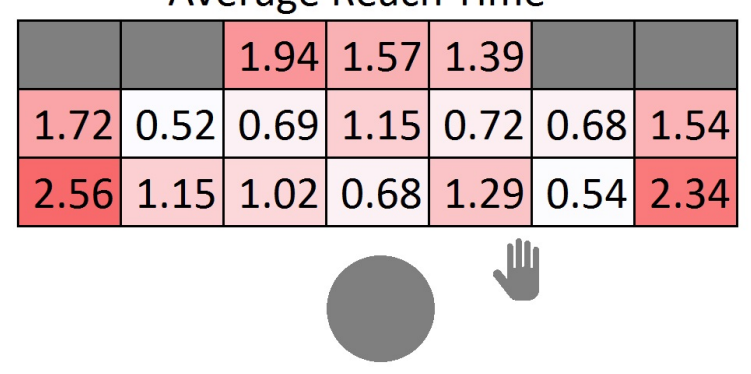

Average Error per Reach

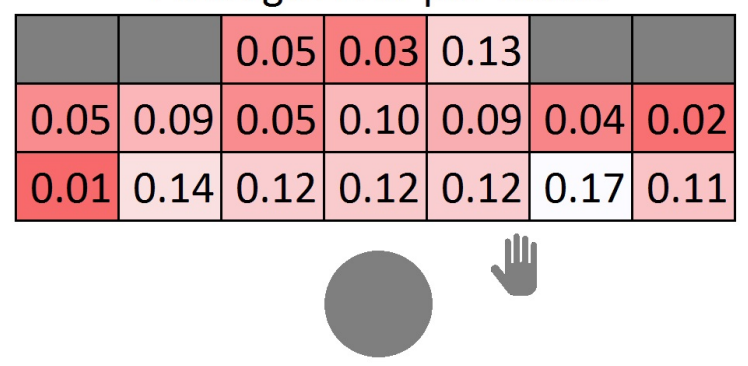

Figure 5.7: Average reach times and errors from Experiment 1 based on table position, where the shape of the data table corresponds to the shape of the physical table, with the user's location represented by the gray circle. Gray cells represent areas of the table not used in the study. 


\subsubsection{Qualitative Feedback}

In addition to the performance results, we gathered qualitative feedback from participants through a semi-structured interview at the end of the study. In terms of overall preference of techniques, 9 participants preferred the translational shift technique, 5 preferring interpolated reach, and 2 felt they were about equal in terms of difficulty of use.

In general, participants did not like tasks with toward offset, where the virtual object seemed visually closer than the physical object actually was. This was especially true for interpolated reach where the hand would move slower due to a small virtual distance and large physical distance; four participants stated this slow motin control made them feel disoriented or nauseated. Most did not mind the interpolated reach tasks with fast interpolation (where the virtual distance exceeded the physical distance). One participant noted that in one subset of tasks with 10 inch offsets during the interpolated reach session, it felt like they were doing one-to-one motion. However, interpolation was more disorienting to a majority of participants, and there was a more obvious disconnect with the physical world as the hand was not moving the way they expected.

The main criticism for translational shift was for offsets that took the virtual hand out of the peripheral view of the user, which caused confusion and required the participant to initially look around for the hand. Several participants commented on being less connected with the hand in translational shift compared to interpolated reach because the hand was constantly being placed in different locations relative to the body. Some related it to controlling a "puppet" or "ghost" hand and did not feel like it was their own. For both techniques, many found they had to rely more on visuals to guide their hand to the object as opposed to their usual intuition and proprioceptive senses; the impression was that the techniques were usable but needed practice over time. The randomness of the tasks and offsets were also noted by some to cause difficulty in adjusting to the techniques. 
Participants felt the haptic prop was beneficial overall, explaining that it made the experience more immersive and gave them a real-world physical cue of when they have the object in their grasp. Some participants noted that being seated at a table that was similar in shape and size to the virtual table made them think about the real world more and how it was different in the virtual world. There was also a fear of hitting or knocking over the bottle during the experiment. One participant suggested that they would probably perform better if the table was larger and the object was smaller to reduce the chances of error. A comment was also made that stretching and reaching across the table was uncomfortable. Some minor visual discrepancies, such as the thumb not being tracked or the hand clipping through parts of the virtual object, brought some participants out of the experience. 


\section{EXPERIMENT 2}

\subsection{Goals and Hypotheses}

Experiment 1 required participants to complete a set of consecutive tasks that were randomly ordered. Each task had a different combination of offset direction, offset size, and physical object table position. The main purpose of experiment 1 was to understand how each of these different independent variables can affect a participant's performance in reaching tasks. However, due to the randomness and unpredictable nature of technique orderings, it was likely difficult for participants to ever adapt to any of the technique configurations. We therefore conducted a second experiment to help understand how one would be able to adapt to continued use of the remapping techniques with a constant offset. Experiment 2 aimed to study how task performance changed over time with the different remapping techniques if participants were given tasks with the same offset effect.

Therefore, in Experiment 2, participants completed multiple reaching tasks, similar to in Experiment 1. However, the difference in Experiment 2 is that each consecutive reaching trial used the same remapping configuration. For the translational shift sessions, all remapped trials used the same offset size and offset direction and for interpolated reach sessions, the virtual bottle was offset relative to the starting hand position and physical bottle position to simulate the same interpolation speed and direction. We hypothesized that performance would improve over time with continued use of the same technique. We also hypothesized that after continued reaching with a remapped technique, it would take time for users to adjust back to normal reaching with one-to-one hand movements.

\subsection{Experimental Task and Environment}

Experiment 2 used the same virtual environment as Experiment 1. However, for Experiment 2 , the virtual table length and width were increased by 1.5 times the original to 
allow for virtual offset positions that would otherwise appear off the table. In this experiment, participants were again asked to reach and grab the virtual object when it changed from red to green and the sign in front of them signaled for them to "Go". However, only the reaching portion of the task was required for this experiment with no target placement after. Once the object was reached, the virtual object and hand would fade away, indicating the successful completion of that task.

\subsection{Experimental Design}

The experiment was designed to first have participants complete multiple consecutive reaching tasks with the same remapping configuration for different object positions. Then, after multiple reaches with a remapped technique, the remapping was disabled, and participants immediately completed additional reaching tasks with normal one-to-one tracked movement.

The experiment followed a repeated-measures design with four technique configurations: (1) translational shift with a 20-inch offset to the user's right, (2) translational shift with 20-inch offset in the forward direction (away from the user), (3) fast interpolated reach, and (4) slow interpolated reach. Note that the interpolated reach technique does not have a fixed offset size because the offset depends on the vector from the participants hand to the physical object. From this vector, the object was either offset 10 inches in the direction of the vector for fast interpolated reach or offset 10 inches in the opposite direction (toward the participant) for slow interpolated reach. The order that participants tested the techniques were balanced using a Latin square. Task order within each technique configuration was randomized for each participant.

As in Experiment 1, the reaching task in Experiment 2 involved different bottle positions on the table. The number of possible locations was reduced from 17 in Experiment 1 to 10 in Experiment 2 because the focus was studying adaption to techniques over time 
rather than to evaluate reaching difficulty and performance of each technique. All participant again started with a session with a normal one-to-one mapping as a baseline for comparing reach times. The one-to-one session consisted of 20 tasks where a task for each of the 10 locations was performed twice. The average of the two one-to-one tasks was used as the base time for that table location.

\subsection{Procedure}

Participants sat at a table with a stationary chair and first completed a consent form. Participants filled out a background questionnaire just as in Experiment 1. Next, the experimenter explained the reaching task, and participants completed a practice session where they had to reach and grab the virtual object with a matched one-to-one mapping five times at varying locations. After the practice session, participants were given a short 5-minute break before starting the main trials.

The main reaching tasks for the study were broken into five sessions. In the first session, all participants performed 20 reaching tasks with one-to-one mapping. In each of the the following four sessions, participants performed 30 consecutive reach tasks. Of these 30 reaches, the first 20 reaches included the offset for one of the four possible technique configurations. Immediately after performing the 20 tasks with the remapping technique, the technique was switched to regular one-to-one mapping for 10 additional reach tasks that covered each of the possible ten table locations. Participants were not notified of the technique change during the sequence of tasks.

In between all sessions, participants were required to take a 5-minute break from the virtual environment. During these breaks, participants were asked to reach for the physical bottle and move it around the desk a few times to help them recalibrate their senses to the real world before moving on to the next session with another remapped technique After finishing all sessions with the different techniques, the experiment was complete. A 
participant's completion of Experiment 2 took approximately 45 minutes.

\subsection{Participants}

Twelve university students ( 5 male, 7 female) took part in Experiment 2 . The participants' ages ranged from 24 to 29 with a median of 26 years. Participants were graduate students in degree programs related to computing, engineering, and art. Eight participants reported spending at least one hour a week playing 3D video games, and all participants reported having prior experience with VR. All participants were required to use their right hand for the experiment. Handedness or hand dominance was not surveyed as all participants' reach times were measured in reference to their own one-to-one reach times.

\subsection{Results and Discussion}

For Experiment 2, results consist of the times taken to reach the target object over the sequence of 30 trials for each of the four technique configurations. Similar to Experiment 1, the times reported are based on the difference between the recorded trial times and the corresponding reach time for the associated table position that were recorded during the first session of 20 normal one-to-one reaching tasks. Because the time reported is based off of a difference, a value of 0 indicates the reach time taken during a trial was the same as the one-to-one reach, and a negative value indicates a faster reach time than that of the initial one-to-one reach time.

Figures 6.1 and 6.2 shows time differences as trials progressed from left to right. It should be noted that regardless of trial number, the times reported are expected to fluctuate at times due to the nature of different table positions being more difficult than others. This fluctuation can be further mitigated in future tests by limiting the number of possible table positions even further to a more localized area of the table. Despite this, the overall results show that reach times did decrease over time with continued use of the remapped techniques over 20 reaches. These trends provide clear evidence that participants were 
adjusting to most techniques over time. Within 20 reaches of the remapped trials, the times did not often reach the baseline zero level. However, it is possible that they would have reached zero with additional practice with the remapping techniques, but we cannot extrapolate based on these results.

An exception to the technique-acclimation effect was observed for the fast interpolated technique, which shows little change over the 20 reaches (see Figure 6.2b). From the results of Experiment 1, we found that users were generally comfortable with fast interpolation, and offsets away from the user saw better performance than towards the user, which may explain the lack of a noticeable improvement over time within 20 tasks. In contrast, with a remapping like for right translational shift, which takes the virtual hand out of the user's field of view, and slow interpolated reach, which may initially catch users by surprise, we see a noticeable improvement in speed from the first few tasks to the last.

When switching back to one-to-one movement for the last 10 reaches (trials 21-30), speed improves further and quickly returns to the zero baseline of normal reaching. Reacclimation to one-to-one motion occurred quickly-with average times returning to the baseline within approximately five reaches. However, the fast interpolation technique again was the exception for this results. Reacclimation to normal took longer after using fast interpolation, and although the mean time does drop close to the baseline, the average did not reach the baseline in the 10 tasks.

Overall, these results suggest that it does take time to adjust to using remapped techniques, and improvements can be achieved even after a short period of practice. Based on these results, it is expected that it would be difficult to change to different remapping approaches in the same application.

Furthermore, the different outcomes with the fast interpolation technique demonstrate that it can be easier or harder to adjust to different remapping techniques. Some techniques may require more practice than others. Additionally, the higher number of trials needed 
to reacclimate to normal reaching suggests it may take longer to "unlearn" remapped motions that are more difficult to learn. We suspect that if a technique is less natural, it will require more attention and conscious effort to effectively control the interaction, and it will therefore take more time to stop using the remapped movement strategy. Also note that participants were not notified of the change from remapped technique back to normal one-to-one motion after the first 20 tasks, so participants could not be sure how the motion would work. It would also be interesting to study normal one-to-one motion in the real world after practicing with the remapped technique (similar to the approach by Bodenheimer et al. [12]). 


\section{Translational - Offset Forward}

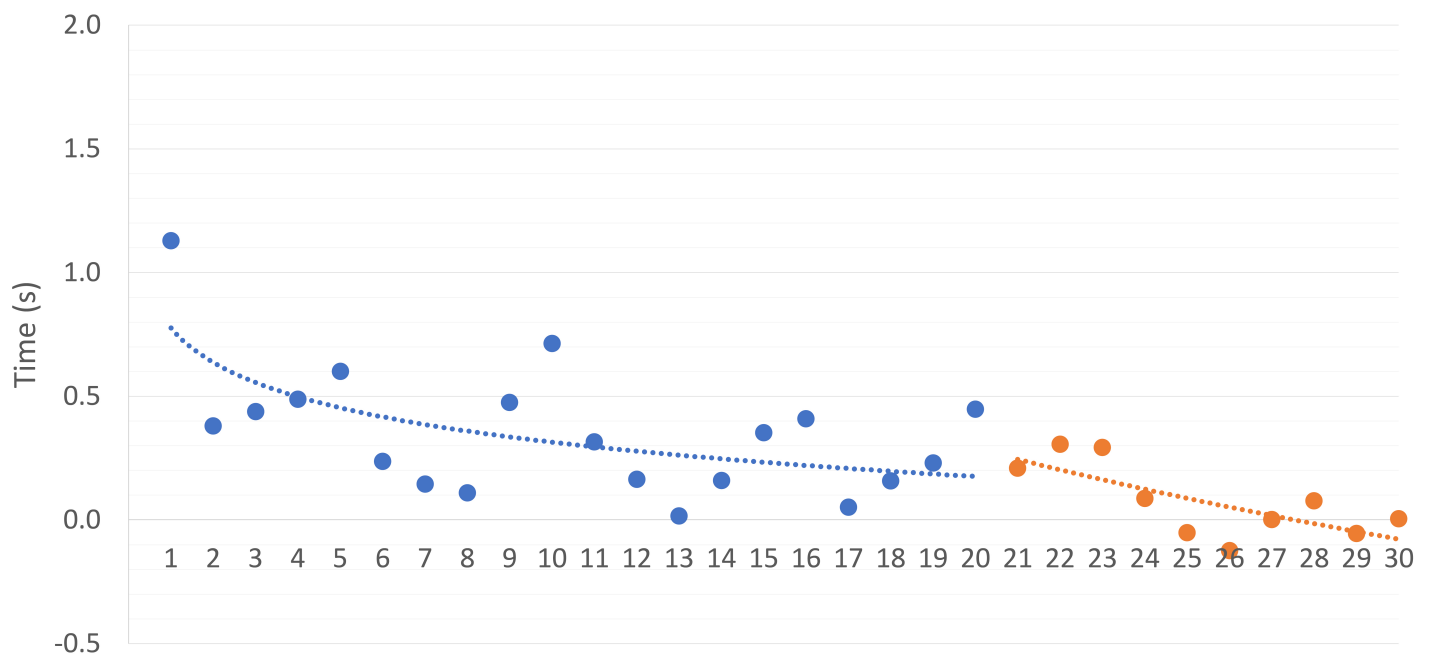

(a)

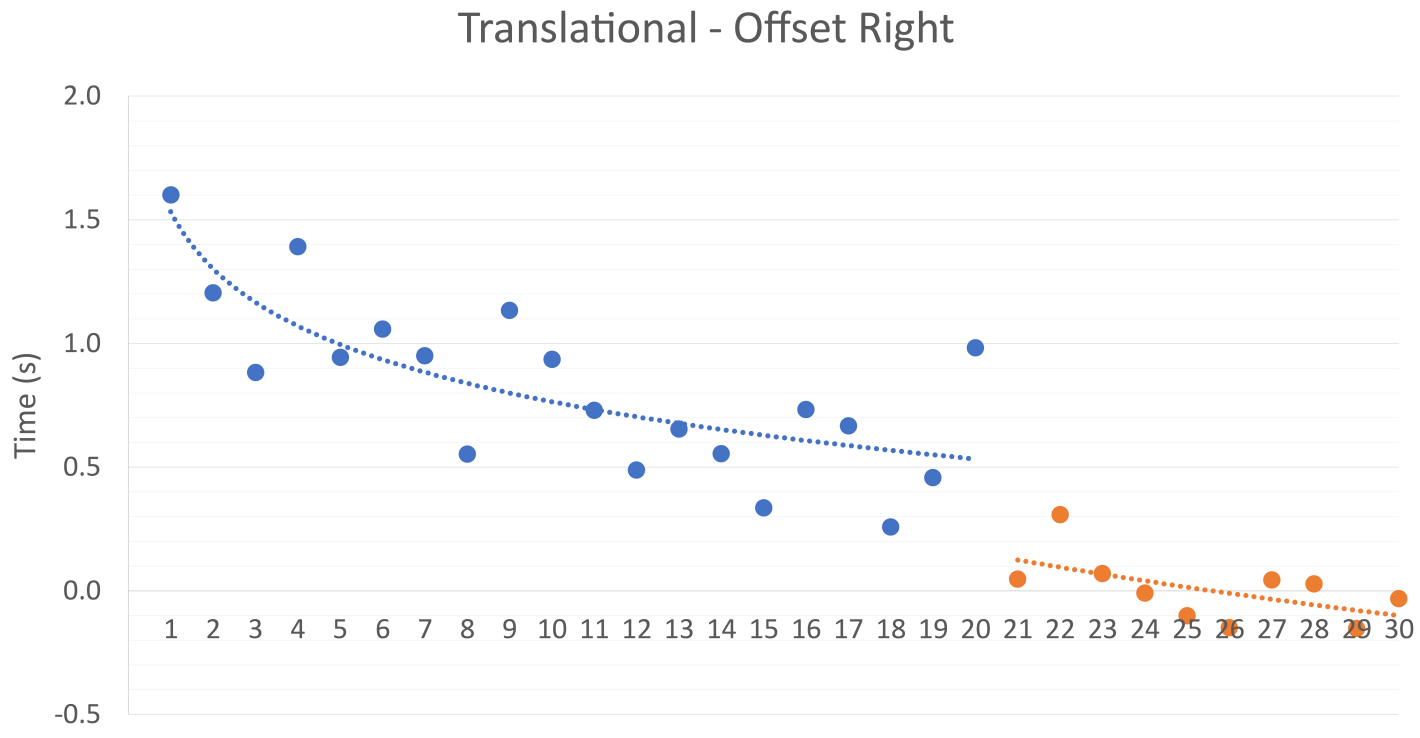

(b)

Figure 6.1: Results for Experiment 2 showing reach time over 30 tasks for translational shift configurations. (a) Translational shift offset forward (b) Translational shift offset right 


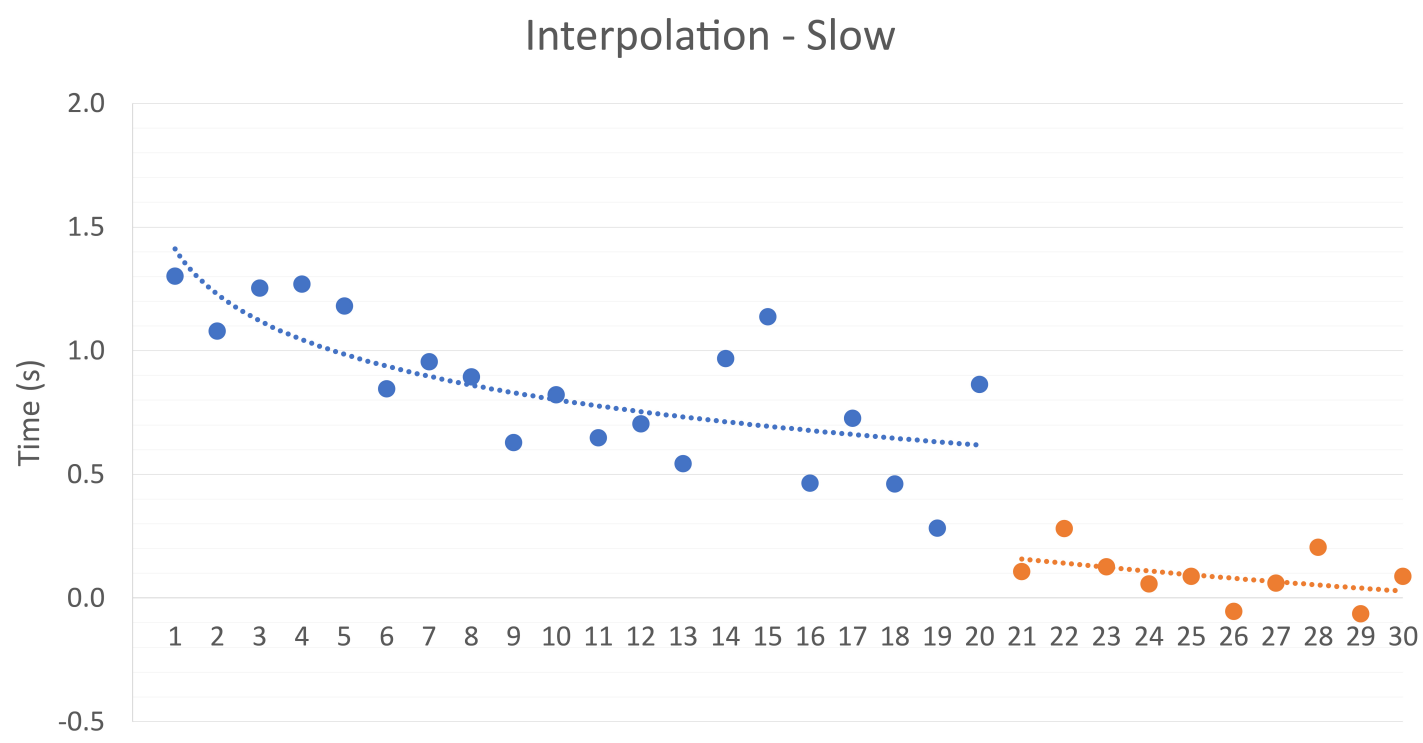

(a)

Interpolation - Fast

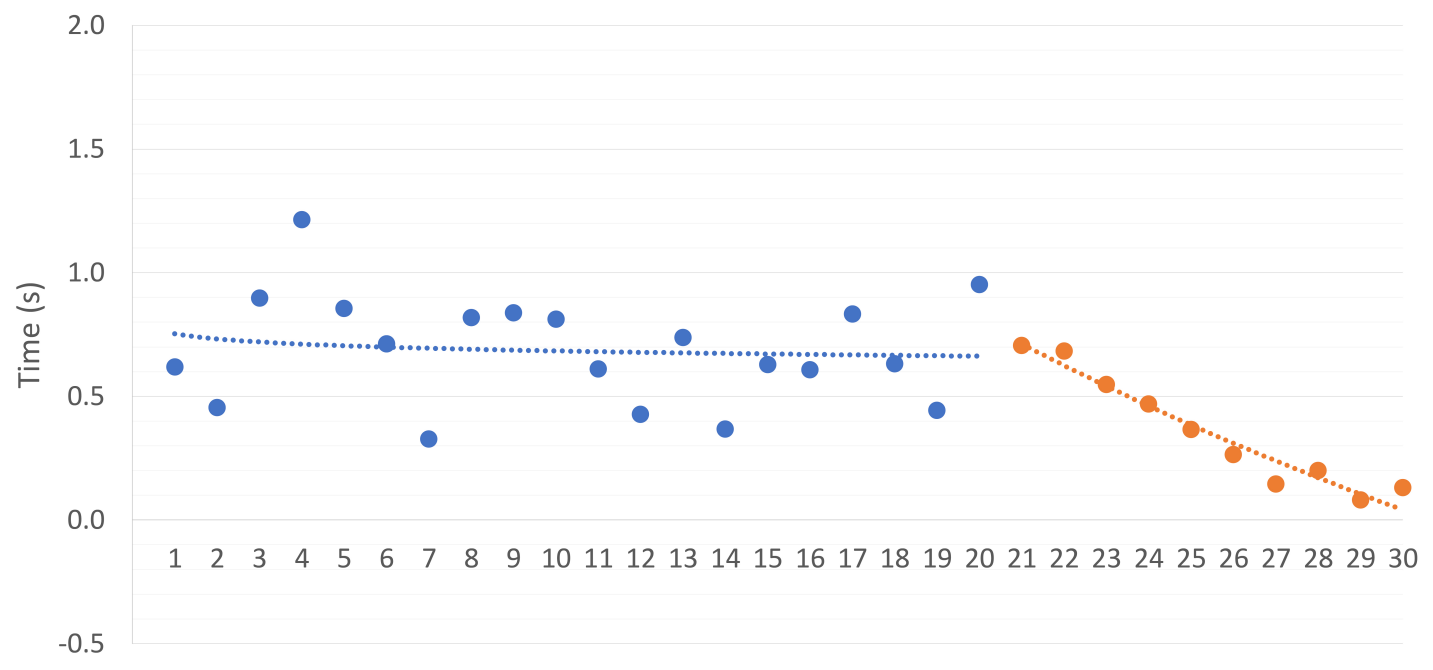

(b)

Figure 6.2: Results for Experiment 2 showing reach time over 30 tasks for inteprolated reach configurations. (a) Slow interpolated reach (b) Fast interpolated reach 


\section{CASE STUDY}

\subsection{Goals}

After the two controlled experiments, we conducted a case study to better understand practical issues for applying the two remapping techniques in VR applications. The case study primarily involved (1) the design and development of an immersive game, and (2) an informal usability evaluation of the game. The game design also demonstrates how the remapped reaching techniques can be used with multiple virtual objects that can dynamically adjust based on gaze direction.

\subsection{Game Application}

For the test application, we created an immersive game with two parts: (1) a weaponscrafting scenario where users mix and match different objects to craft weapons, and (2) a monster combat scenario where the user must use the crafted weapons to defeat various creatures. Figures 7.1 and 7.2 show screenshots from the game. The game takes place in a forest environment populated with trees and rocks, and the player is seated at a virtual workbench that aligns with the physical table. The game was developed with assets from the Unity Asset Store.

The controls for the game required the user only to reach, grab, and move objects with their hands. Object selection was done using user gaze based on the view orientation (similar to as in [17]). Players had control of only their tracked right hand to interact with the virtual objects. Selected objects were highlighted with a green outline to indicate which object was the currently selected for interaction. The selected object was locked once the hand was no longer close to the body to prevent accidental offset switching during reaching. In order to select a new object, players needed to retract their hand to the armrest or close to their body to allow the gaze selection to be re-enabled. 


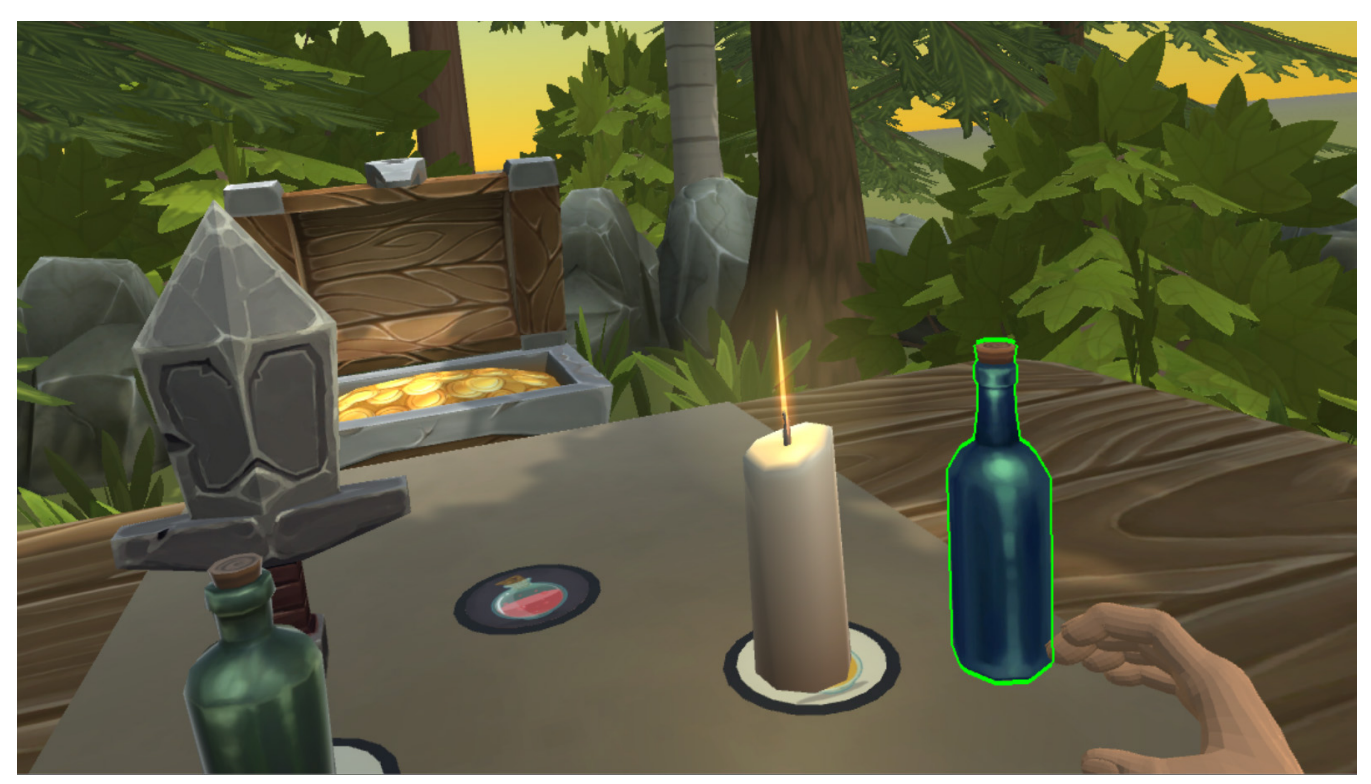

Figure 7.1: The virtual hand is offset to map to the virtual object highlighted in green based on user gaze.

Instructions for the game were presented at the start of the application, and they explain the player must craft weapons to defend themselves from the creatures that live in the woods. For the weapon crafting section of the game, the player is presented with four interactive objects: a sword, a candle, a blue bottle, and a green bottle. In order to craft weapons, the player must grab objects and place them on three different visual markers on the workbench. In order to proceed with the game, the player must combine the four ingredients in all possible combinations of three, which leads to a total of four possible weapons to craft. Each weapon is seen hovering in the background once crafted.

Once all four weapons are discovered, the next phase of the game starts immediately, and a monster appears in front of the player. The player is then able to select any of the four weapons that they created using gaze selection. Once the player grabs a weapon, the player can then swing it at the creature to attack it (as shown in Figure 7.2). Each creature can only be damaged by a specific weapon, so the player must find which weapon 


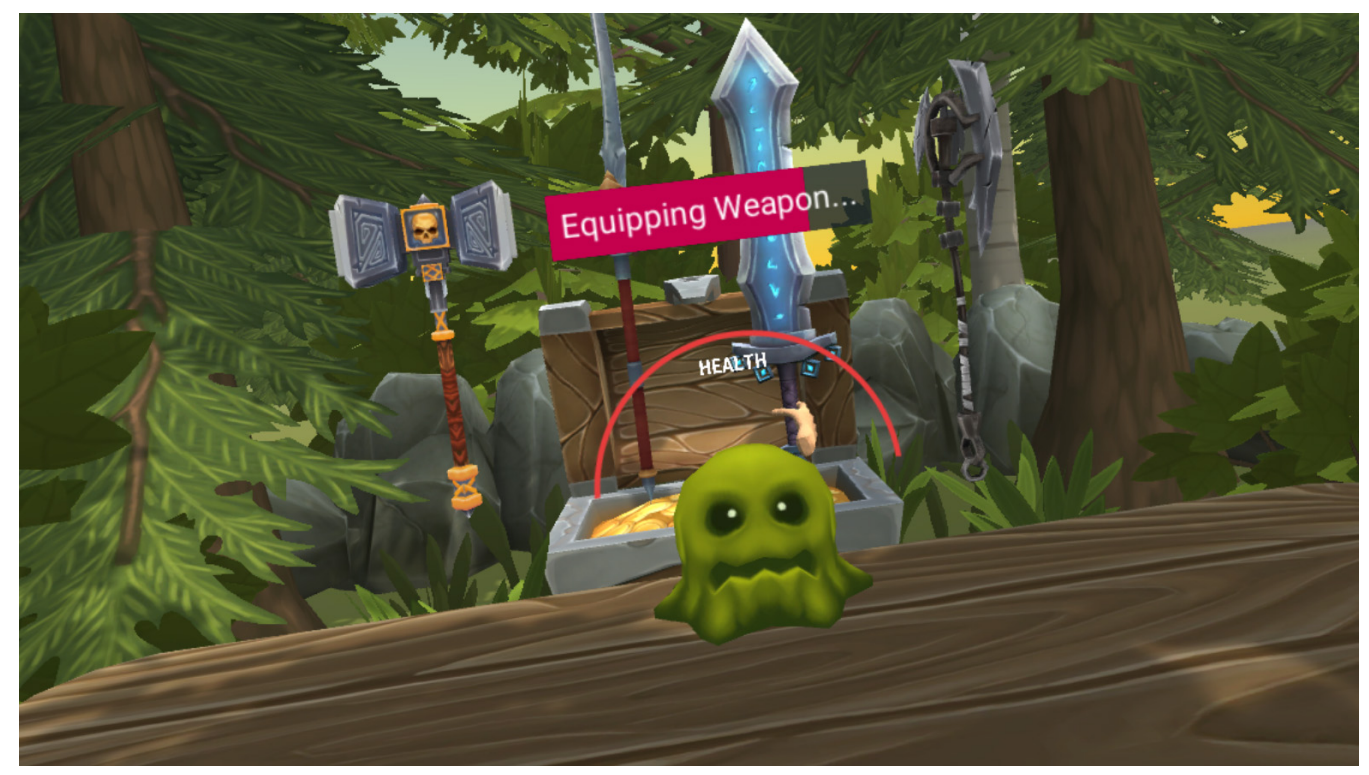

(a) Users select one of the weapons that were crafted in the first part of the game

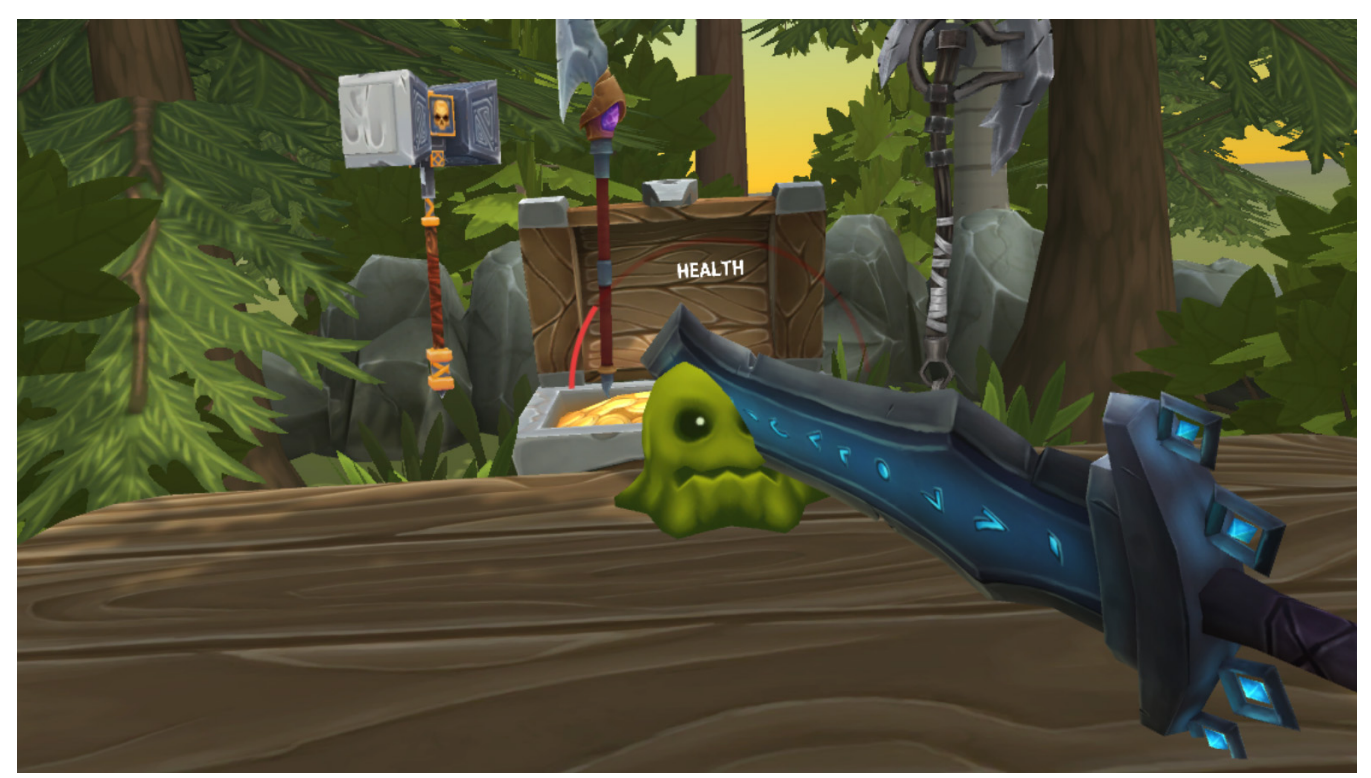

(b) Weapon and hand are returned to default position to allow the user to attack the monster

Figure 7.2: Monster combat scenario 
is effective on the specific enemy. Monsters have a health bar indicating current health and amount of damage taken, and if the weapon is ineffective, the health bar will flash without depleting. Once all four enemies are defeated, the player has successfully completed the game.

\subsection{Usability Evaluation}

We conducted a small usability evaluation of the game using the translational shift and interpolated reach techniques. Four participants (two male, two female) played through the game twice where each session used a different remapping technique. Two participants started with translational shift while the other two participants started with interpolated reach. The study was conducted using the same setup as the two experiments with the same VR system, table, chair, and physical prop. All four participants had previously experienced the remapping techniques from the previous experiments. However, other than the written instructions presented in-game, participants were not given any instructions or explanations of how to play the game or what to expect.

The participants played through both the weapon crafting and monster battling portions of the game and took a short break between the two play sessions. Participants were encouraged to express their thoughts while playing the game and ask questions if they were stuck or confused about game mechanics. After completing both sessions, a semistructured interview was conducted to collect qualitative feedback from the participant on how they felt about the game and their thoughts on using the two different techniques. Each playthrough took approximately 5 to 10 minutes to complete. The second session was always faster than the first since the game was identical for both sessions with the exception of the technique being used, so participants understood the goal of the game by then. 


\subsection{Results and Design Guidelines}

From implementing the hand remapping techniques in a game application, we came across some unique technical hurdles that did not come up in the experimental scenarios and should be addressed in applications using remapping techniques. One of the main challenges was how to handle object selection with multiple virtual objects. We opted to keep the entire experience hands-free with no controllers so the entire experience would only require physical hand and head interaction. Because object selection was based solely on head orientation, user who prefer to move their eyes to look at objects rather than adjust their head orientation might have difficulty or annoyance with achieving accurate selection. Eye tracking could be an improvement for this and limit the need for additional head and neck movement. Object occlusion is also an issue for gaze-based selection.

With regard for the remapping techniques, the main challenge for translational shift was to find an unobtrusive way to transition between offset changes when the user changed target selections. We were initially concerned that abrupt changes to the position of the virtual hand would be jarring or confusing for participants. Fading the hand in and out between updates worked reasonably well, but additional exploration of other options would be interesting for future work.

For interpolated reach, one of the biggest problems was dealing with large offsets when reaching distant objects such as hovering weapons in the game (such as the hammer in the left half of Figure 7.2a). Because of the linear nature of the hand movements with the interpolated reach technique, movements away from the intended path were sensitive to remapping. This could be addressed in future iterations of the technique by using an easing function for more gradual interpolation near the hand's starting and ending locations.

Reducing the overall learning curve to play the game is something that should be addressed as well. Participants came in to the study assuming they could interact with the 
virtual objects similarly to the real world where they can move their hand from one object to another. Participants took some time to realize they needed to bring their hand back to select a new object, which is a limitation of common implementations of remapping techniques (e.g., [16]). More descriptive instructions, visual cues, or practice could help with this problem.

Finally, because users were manipulating multiple virtual objects in the same virtual space, a physical object could end up far from the user or be placed off the physical table depending on the order of selecting and moving objects. This is a major concern for making remapped reach viable for multiple virtual objects using a single passive-haptic prop. Potential solutions include: taking advantage of change blindness to allow the environment and virtual objects move to more convenient locations when they are not in view (similar to [20]), teleporting the user to a more appropriate view that would reduce difficult reaches or object placement (as in [21]), or providing additional visual cues and warnings. Another possibility is further adjusting the control-display ratio to scale virtual movements such that physical objects could remain within a desirable physical range. 


\section{DISCUSSION \& CONCLUSION}

\subsection{Summary}

We evaluated two remapping techniques that allow hand-reaching for selection and manipulation of virtual objects using a passive-haptic props. Our findings demonstrate that remapping techniques can work for use with reach, but there are clear performance implications in certain cases. Overall, the translational shift implementation was generally superior to interpolation techniques for the cases tested in our research.

In Experiment 1, we compared the translational shift and interpolated reach remapping techniques and compared their performance under various conditions in terms of time and errors, and Experiment 2, we studied acclimation time to adjust using the modified reach techniques. Experiment 1 considered performance implications of different offset sizes, offset direction, and physical object locations. Overall, the translational shift technique performed better than interpolated reach and proved to be more robust for situations with larger mismatches between virtual and physical objects. No significant performance penalties were detected with between offsets of 10 and 14 inches for hand reaching, demonstrating that people can generally tolerate some mismatches between virtual and physical content.

Direction of offset was found to have a significant effect on reach time, where virtual objects that closer toward the user were shown to be slower. Larger offsets (30 inches) were found to also be significantly slower than all other offset sizes, and the use of translational shift with offsets away from the user were the most tolerable to larger offsets for reach performance. Our results also indicate that reaching errors increased with interpolated reach using larger offsets as opposed to translational shift, where errors occurred less frequently and more consistently across situations with different offset sizes. Unsur- 
prisingly, the results also show that objects positioned farther from a user can produce more error and longer reach times when the virtual hand is remapped to accommodate mismatch.

Feedback from participants generally match the performance results, showing a preference for translational shift from a majority of participants. However, translational shift had problems with participants needing to look for objects and feeling a lack of ownership of the virtual hand. Still, translational shift was considered more comfortable overall, and several participants commented on the disorienting nature of interpolated reachespecially when the virtual hand moved slower than expected.

The acclimation results from Experiment 2 show that, with practice, users can adjust to remapped motions to improve proficiency. These results suggest that use of modified reach techniques might become easier or feel more natural over time with continued use. On the other hand, users did not adjust to all techniques the same way, and not all participants acclimated to the remapped techniques to the point that reach times reached one-to-one speeds within the scope of the study. In addition, fast interpolation showed noticeable acclimation problems when compared to the other remapping techniques. We also tested for reacclimation to normal one-to-one mapping after using the remapped techniques, and results showed that after using fast interpolated reach, it took longer to reacclimate back to normal hand movements.

In addition, we presented a case study in the design and development of an immersive game using the remapped reach techniques, and we discussed several practical implications. For use of a single passive-haptic prop with multiple virtual objects and dynamic offset changes during a continued experience, there are challenges to address in terms of object selection and keeping the physical object within physical reach and within bounds of a physical surface area.

Overall, the results of the two experiments and case study demonstrate that remapped 
reaching with passive-haptic props is a promising approach for enabling realistic tactile feedback for direct hand interaction, but considerations for technique limitations is important for avoiding substantial penalties to performance, comfort, and usability.

\subsection{Future Research}

In future research we would like to understand further how these remapping techniques can be utilized in practical settings for VR applications. The case study presented in this thesis raises several questions on how we can make these techniques usable. A major concern is how to keep physical props within a user's reach. Uncomfortable reaches can work against the application and bring users out of the experience so further studies should revolve around making remapping reach as seamless and comfortable as possible. One of the driving motivations for this research involving haptic props is the cost and accessibility for VR consumers so future research should focus on cost effective and practical solutions for providing seated virtual reality experiences with haptic feedback. 


\section{REFERENCES}

[1] B. E. Insko, M. Meehan, M. Whitton, and F. Brooks, Passive haptics significantly enhances virtual environments. $\mathrm{PhD}$ thesis, University of North Carolina at Chapel Hill, 2001.

[2] Z. He, F. Zhu, A. Gaudette, and K. Perlin, "Robotic haptic proxies for collaborative virtual reality," arXiv preprint arXiv:1701.08879, 2017.

[3] E. A. Suma, Z. Lipps, S. Finkelstein, D. M. Krum, and M. Bolas, "Impossible spaces: Maximizing natural walking in virtual environments with self-overlapping architecture," IEEE Transactions on Visualization and Computer Graphics, vol. 18, no. 4, pp. 555-564, 2012.

[4] S. Razzaque, Z. Kohn, and M. C. Whitton, "Redirected walking," in Proceedings of EUROGRAPHICS, vol. 9, pp. 105-106, Citeseer, 2001.

[5] E. Burns, S. Razzaque, A. T. Panter, M. C. Whitton, M. R. McCallus, and F. P. Brooks, "The hand is slower than the eye: A quantitative exploration of visual dominance over proprioception," in Virtual Reality, 2005. Proceedings. VR 2005. IEEE, pp. 3-10, IEEE, 2005.

[6] R. J. Stone, "Haptic feedback: A brief history from telepresence to virtual reality," in Haptic Human-Computer Interaction, pp. 1-16, Springer, 2001.

[7] W. A. McNeely, "Robotic graphics: A new approach to force feedback for virtual reality," in Virtual Reality Annual International Symposium, 1993., 1993 IEEE, pp. 336-341, IEEE, 1993.

[8] I. Poupyrev, N. Tomokazu, and S. Weghorst, "Virtual notepad: handwriting in immersive vr," in Virtual Reality Annual International Symposium, 1998. Proceedings., 
IEEE 1998, pp. 126-132, IEEE, 1998.

[9] E. Ebrahimi, B. Altenhoff, L. Hartman, J. A. Jones, S. V. Babu, C. C. Pagano, and T. A. Davis, "Effects of visual and proprioceptive information in visuo-motor calibration during a closed-loop physical reach task in immersive virtual environments," in Proceedings of the ACM Symposium on Applied Perception, pp. 103-110, ACM, 2014.

[10] I. Poupyrev, M. Billinghurst, S. Weghorst, and T. Ichikawa, "The Go-Go interaction technique: Non-linear mapping for direct manipulation in VR," in Proceedings of the 9th annual ACM symposium on User interface software and technology, pp. 79-80, ACM, 1996.

[11] I. Poupyrev, T. Ichikawa, S. Weghorst, and M. Billinghurst, "Egocentric object manipulation in virtual environments: empirical evaluation of interaction techniques," in Computer graphics forum, vol. 17, pp. 41-52, Wiley Online Library, 1998.

[12] B. Bodenheimer, S. Creem-Regehr, J. Stefanucci, E. Shemetova, and W. B. Thompson, "Prism aftereffects for throwing with a self-avatar in an immersive virtual environment," in Virtual Reality (VR), 2017 IEEE, pp. 141-147, IEEE, 2017.

[13] L. Kohli, M. C. Whitton, and F. P. Brooks, "Redirected touching: training and adaptation in warped virtual spaces," in 3D User Interfaces (3DUI), 2013 IEEE Symposium on, pp. 79-86, IEEE, 2013.

[14] L. Kohli, "Redirected touching: Warping space to remap passive haptics," in $3 D$ User Interfaces (3DUI), 2010 IEEE Symposium on, pp. 129-130, IEEE, 2010.

[15] L. Kohli, M. C. Whitton, and F. P. Brooks, "Redirected touching: The effect of warping space on task performance," in 3D User Interfaces (3DUI), 2012 IEEE Symposium on, pp. 105-112, IEEE, 2012. 
[16] M. Azmandian, M. Hancock, H. Benko, E. Ofek, and A. D. Wilson, "Haptic retargeting: Dynamic repurposing of passive haptics for enhanced virtual reality experiences," in Proceedings of the 2016 CHI Conference on Human Factors in Computing Systems, pp. 1968-1979, ACM, 2016.

[17] L.-P. Cheng, E. Ofek, C. Holz, H. Benko, and A. D. Wilson, "Sparse haptic proxy: Touch feedback in virtual environments using a general passive prop," in Proceedings of the 2017 CHI Conference on Human Factors in Computing Systems, pp. 37183728, ACM, 2017.

[18] E. Schmider, M. Ziegler, E. Danay, L. Beyer, and M. Bühner, "Is it really robust?," Methodology, 2010.

[19] M. W. Vasey and J. F. Thayer, "The continuing problem of false positives in repeated measures anova in psychophysiology: A multivariate solution," Psychophysiology, vol. 24, no. 4, pp. 479-486, 1987.

[20] E. A. Suma, S. Clark, D. Krum, S. Finkelstein, M. Bolas, and Z. Warte, "Leveraging change blindness for redirection in virtual environments," in Virtual Reality Conference (VR), 2011 IEEE, pp. 159-166, IEEE, 2011.

[21] M. Suhail, S. P. Sargunam, D. T. Han, and E. D. Ragan, "Redirected reach in virtual reality: Enabling natural hand interaction at multiple virtual locations with passive haptics," in 3D User Interfaces (3DUI), 2017 IEEE Symposium on, pp. 245-246, IEEE, 2017. 
APPENDIX A

EXPERIMENT DOCUMENTS 


\section{Background Questionnaire}

${ }^{*}$ Required

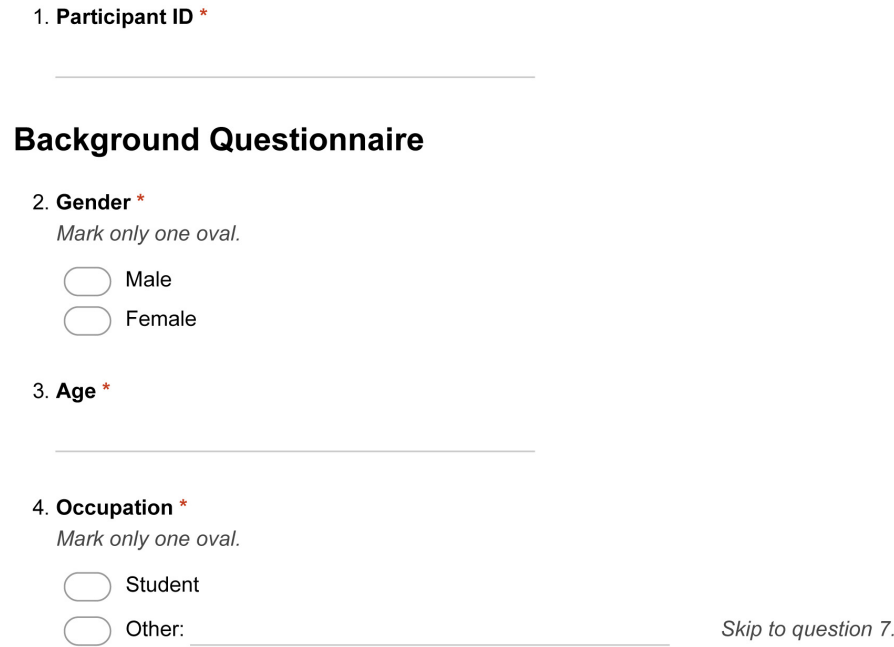

\section{Student Information}

5. Major / Degree Specialization *

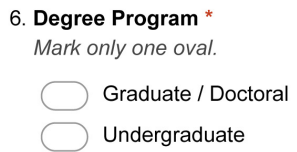

\section{User Usage}

7. Approximately how many hours a week do you use computers? *

Figure A.1: Background Questionnaire Page 1. 
8. Please rate your knowledge of computers and technology based on how you think you compare to the general population *

Mark only one oval.

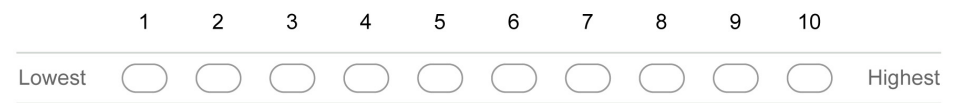

9. Please rate your experience with computer games *

Mark only one oval.

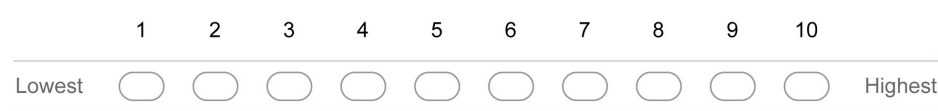

10. Briefly Explain?

11. Approximately how many hours a week do you play any video games?*

12. Approximately how many hours a week do you play 3D video games? (For Example, FPS or third-person RPG's games) *

13. Please rate your experience with virtual reality * Mark only one oval.

$\begin{array}{lllllllllll} & 2 & 3 & 4 & 5 & 6 & 7 & 8 & 9 & 10 & \\ \text { Lowest } \square & \square & \square & \end{array}$

14. Briefly Explain?

Figure A.2: Background Questionnaire Page 2. 